\title{
An operational epidemiological model for calibrating agent-based simulations of pandemic influenza outbreaks
}

\author{
D. Prieto • T. K. Das
}

Received: 6 April 2013 / Accepted: 12 February 2014 / Published online: 8 April 2014

(C) Springer Science+Business Media New York 2014

\begin{abstract}
Uncertainty of pandemic influenza viruses continue to cause major preparedness challenges for public health policymakers. Decisions to mitigate influenza outbreaks often involve tradeoff between the social costs of interventions (e.g., school closure) and the cost of uncontrolled spread of the virus. To achieve a balance, policymakers must assess the impact of mitigation strategies once an outbreak begins and the virus characteristics are known. Agent-based $(\mathrm{AB})$ simulation is a useful tool for building highly granular disease spread models incorporating the epidemiological features of the virus as well as the demographic and social behavioral attributes of tens of millions of affected people. Such disease spread models provide excellent basis on which various mitigation strategies can be tested, before they are adopted and implemented by the policymakers. However, to serve as a testbed for the mitigation strategies, the $\mathrm{AB}$ simulation models must be operational. $\mathrm{A}$ critical requirement for operational $\mathrm{AB}$ models is that they are amenable for quick and simple calibration. The calibration process works as follows: the $\mathrm{AB}$ model accepts information available from the field and uses those to update its parameters such that some of its outputs in turn replicate the field data. In this paper, we present our epidemiological model based calibration methodology that has a low computational complexity and is easy to interpret. Our model
\end{abstract}

\footnotetext{
D. Prieto $(\bowtie)$

Department of Industrial and Manufacturing Engineering,

Western Michigan University, 1903 W. Michigan Ave.,

Kalamazoo, MI 49008-5336, USA

e-mail: diana.prieto@wmich.edu

T. K. Das

Department of Industrial and Management Systems Engineering, University of South Florida, Tampa, FL 33620, USA

e-mail: das@usf.edu
}

accepts a field estimate of the basic reproduction number, and then uses it to update (calibrate) the infection probabilities in a way that its effect combined with the effects of the given virus epidemiology, demographics, and social behavior results in an infection pattern yielding a similar value of the basic reproduction number. We evaluate the accuracy of the calibration methodology by applying it for an $\mathrm{AB}$ simulation model mimicking a regional outbreak in the US. The calibrated model is shown to yield infection patterns closely replicating the input estimates of the basic reproduction number. The calibration method is also tested to replicate an initial infection incidence trend for a H1N1 outbreak like that of 2009.

Keywords Pandemic influenza model - Operational model calibration Seasonal influenza model

\section{Introduction}

Exact epidemiological features of an emerging pandemic in- fluenza (PI) virus are largely unpredictable. Each PI outbreak in the 20th and the 21 st centuries, so far, has resulted from an antigenic shift, where genetic segments from an influenza virus were substituted by segments of other influenza viruses within human or animal cells [45]. An antigenic shift can only be recognized once symptomatic cases are detected by the surveillance system. However, even after the features of a new pandemic influenza virus are identified, the spread of the virus may be hard to predict. This is due to the fact that a PI virus can co-circulate with other seasonal influenza viruses via coinfection (where individuals are simultaneously infected by several viruses) or re-infection (where individuals are successively infected by several viruses). Such interactions 
create different immune responses in different individuals [35]. Moreover, PI viruses often evolve genetically while they co-circulate with other influenza viruses [42, 43]. Such uncertainties of an emerging PI virus have always baffled the public health policy makers.

In recent years, several research groups have dedicated their efforts to develop PI models intended to reduce the uncertainties of mitigation decision making during pandemic outbreaks. Agent-based (AB) simulation models have been presented in the literature as a main decision support tool to predict the expected local and global impact of a likely future pandemic, and to test the performance of mitigation strategies in various outbreak scenarios. AB simulation models have been used to test combinations of mass-action and individual-based strategies in small localities [29, 36, 58], urban areas [33], rural areas [24, 37], countries $[9,16,23,27,30]$, and intercontinental areas [10, 11, 25, 34, 38, 39]. Results derived from AB models have been used to inform national scientific committees [4] and draft national preparedness guidelines [3].

Though existing AB models are generally perceived to be useful in supporting public health policymakers (PHP) [52], these models may be further enabled to support PHP with operational decision updates every 4-6 h during PI outbreaks [6]. Such decision updates require that the AB models be populated with fresh data, recalibrated, rerun, and reinterpreted. Most of these steps in existing $\mathrm{AB}$ models entail significant human intervention and require computation times that are longer than the desired operational decision cycles [52].

Enabling $\mathrm{AB}$ models to shorter operational decision cycles thus would require: 1) fast access to the demographic data from the outbreak region, 2) frequent update of social behavioral parameters, 3) more frequent (e.g., daily or weekly) collection of epidemiological data, and 4) quick translation of these data into the model parameters (referred to as model calibration).

Finally, the computational time needed to run the replicates of an AB model and to visualize model outputs should also be reduced to a minimum, which may require the use of high performance computing and novel visual interfaces. A review of the operational features of the existing $\mathrm{AB}$ models and their data sources can be found in [50].

Demographic data in the U.S. is readily available in the form of a synthetic population database, which contains geographically located households and household members. It also includes group quarters, workplace and school locations, and their assigned population members. These information can be used by the $\mathrm{AB}$ models directly without any further translation [5].

Social-behavioral parameters of the AB models must also be updated, since the population affected by the outbreak change their behavioral response to risk as they receive new information about the pandemic. Social behavioral parameters include percentage of infections being reported and rates of compliance to treatment and interventions. $\mathrm{AB}$ models allow updates of the percentages and rates of compliance which could vary with time, with subject, and level of dissemination of the pandemic status. In Europe, Influenzanet weekly collects information about healthcare seeking behaviors using an internet based survey [2, 14]. To our knowledge, no other behavioral data is collected periodically.

Epidemiological data for influenza like illnesses (ILI) is reported weekly in Europe by Influenzanet, and world-wide by Google Flutrends [2, 28]. The Flutrends data by Google is the only open access data [1]. However, a challenge that remains is the unavailability of frequent updates of the virus confirmed cases, with necessary statistical validity, to support estimation of reliable time series on the overall pandemic cases. Such a time series can be used either directly to fit the internal parameters of $\mathrm{AB}$ models, or indirectly to estimate classical transmissibility parameters (like the basic reproduction number $R_{o}$ ). Both the time series and the classical parameters can be used to calibrate the internal parameters (e.g., probabilities of infection of contacts). Many of the existing AB models use estimated values of $R_{O}$ as a means to calibrate their internal parameters with the field epidemiological data $[9,15,23,27,29,30,32,33,36$, $37,53,55,58]$. This approach to calibration is justified by the facts that $R_{O}$ is widely recognized to be a measure of disease trend by the community of theoretical epidemiologists [56], that there are several methods for estimating $R_{o}$, and that it has been extensively used to characterize the transmission potential of previous pandemic outbreaks [12, 19, 23, 24, 26, 40].

Many $\mathrm{AB}$ simulation models use human-assisted calibration $[15,29,32,36,37,53]$, while some perform algorithm-driven calibration [44, 58]. Although algorithmdriven calibration is automatic, the speed of the calibration process depends on the complexity of the algorithm (which is not constant for the current algorithms) and also on the nature of the computational resources available to run the model. There exists a body of literature that tries to minimize the number of replicates needed for calibration, using emulators of the complex models and quick search procedures [47], but these methods have not been used for the calibration of epi parameters in pandemic $\mathrm{AB}$ models.

Proper calibration of the $\mathrm{AB}$ models is essential since the public health policymakers are more likely to adopt recommendations if their interpretation of the results from the baseline $\mathrm{AB}$ model matches their understanding of the ground truth. Policymakers tend to prefer models that are capable of abstracting the essential elements of the reality with least possible complexity. They should be able to agree with model assumptions and gain intuition from 
model results without pre exposure to modeling techniques [6-8, 52].

The primary contribution of this paper is an epidemiological (epi) model-based methodology that is capable of automatically calibrating the internal infection probabilities of $\mathrm{AB}$ simulation models using estimated values of $R_{o}$ derived from field data. We show that the calibration process has a constant computational complexity $O(1)$, and it is easy to interpret and implement by the PHP. We test the efficacy of our calibration methodology by embedding the epi model within an $\mathrm{AB}$ simulation model, which mimicks a pandemic influenza outbreak in a large county (with a population of 1.1 million) within the State of Florida in the U.S. We further demonstrate the utility of our methodology by using it to reproduce the initial trends of disease spread data, which was collected during the 2009 H1N1 outbreak in Florida. Finally, we show that our methodology can also support PHP during pandemic influenza outbreaks in the presence of a co-circulating seasonal flu virus.

The paper is organized as follows. In Section 2, we present the epi model based calibration methodology. In Section 3, we present the implementation of the epi model within an AB simulation testbed. Sections 4 and 5 present performance of our calibration methodology and conclusions, respectively.

\section{Epi model-based methodology for AB model calibration}

Let $N_{t}^{i}$ denote the number of individuals infected by a single infectious individual $i$ between time $t$ and $t+1$. Thus, $R^{i}=\sum_{t=0}^{\infty} N_{t}^{i}$ is the total number of infected cases produced by the $i$ th individual, which is referred to as the reproduction number [56]. We use $\mathscr{N}_{t}$ to denote the random variable for the number of new infections created by an individual between time $\mathrm{t}$ and $\mathrm{t}+1$ and $\mathscr{R}$ to denote the random variable for the total number of infections by an individual. Note that $\mathscr{N}_{t}$ and $\mathscr{R}$ are defined over the sample spaces containing $N_{t}^{i}$ and $R^{i}$ for all $i$, respectively, in the population.

Let $I^{k}$ denote the set of infected cases in the $k$ th generation. Then, $I^{0}$ would denote the first set of infected cases that begin an outbreak when the entire population is susceptible. Note that $I^{1}$ would include all cases infected by those belonging to $I^{0}$. Let $\mathscr{R}^{k}$ denote the random variable defined over the sample space containing $R^{i}$, for all $i \in I^{k}$.

Then $E\left[\mathscr{R}^{0}\right]$, for generation $k=0$, is called the basic reproduction number and is commonly denoted in the literature by $R_{O}$ [22]. In population based models (e.g., DE models) the $E\left[\mathscr{R}^{k}\right]$ is an epidemic treshold parameter, meaning that if the value of $E\left[\mathscr{R}^{k}\right]$ is higher than one, an outbreak is in the expansion phase, whereas a value of less than one indicates a contracting phase and an outbreak is considered to be under control.

Let $T^{i}$ denote the disease generation interval of infected individual $i$, i.e., the time interval between the infection of individual $i$ and the infection of a secondary case produced by that individual. We use $\mathscr{T}$ to denote the random variable for the disease generation interval. Note that $\mathscr{T}$ is defined over the sample space containing $T^{i}$ for all $i$ in the population. With the notation established above, the probability of creating a secondary infection by the $i$ th infected individual between time $t$ and $t+1$ can be expressed as $\frac{N_{t}^{i}}{R^{i}}$. Let $w(t)$ denote the probability mass function for the disease generation interval $\mathscr{T}$. Then we have that

$w(t)=\frac{E\left[\mathscr{N}_{t}\right]}{E[\mathscr{R}]}$.

Also, it has been empirically shown in $[17,24]$ that $w(t)$ can also be expressed in terms of the absolute (not logarithmic) viral titer count $V_{t}^{i}$ for individual $i$ between time $t$ and $t+1$. The viral titer count measures the viral load within an individual (e.g., see Table 3 for the daily titer count in cases infected with H1N1). Let the viral titer count random variable be $\mathscr{V}_{t}$ defined over the sample space containing $V_{t}^{i}$, for all $i$ in the population. Then we can write that

$w(t)=\frac{E\left[\mathscr{V}_{t}\right]}{E\left[\sum_{t=0}^{\infty} \mathscr{V}_{t}\right]}$.

We note that calculating $w(t)$ from Eq. 1 is impractical due to the difficulty of identifying the individuals infected by a single case. However, since aggregated values for viral shedding profiles of human volunteers are available for influenza [17], the expression (2) offers a more feasible method to calculate $w(t)$. From Eqs. 1 and 2, we have that

$E\left[\mathscr{N}_{t}\right]=E[\mathscr{R}] \frac{E\left[\mathscr{V}_{t}\right]}{E\left[\sum_{t=0}^{\infty} \mathscr{V}_{t}\right]}$.

Thus, the number of infected cases between time $t$ and $t+1$ is a proportion of $E[\mathscr{R}]$, determined by the viral shedding profile. In what follows, we use Eq. 3 to obtain an expression for the probability of a contact getting infected.

We assume that $\mathscr{N}_{t}$ is binomially distributed with parameters $c_{t}$ and $p_{t}$ (denoted henceforth as $\mathscr{N}_{t} \sim \operatorname{bin}\left[c_{t}, p_{t}\right]$ ), where $c_{t}$ is the number of contact events that an infected case makes between time $t$ and $t+1$, and $p_{t}$ is the probability that a contact becomes infected. From the binomial assumption, we can write that $E\left[\mathscr{N}_{t}\right]=c_{t} p_{t}$, which using Eq. 3 yields

$p_{t}=\frac{E[\mathscr{R}] \frac{E\left[\mathscr{V}_{t}\right]}{E\left[\sum_{t=0}^{\infty} \mathscr{\mathscr { V }}_{t}\right]}}{c_{t}}$.

The binomial assumption implies that during time $t$, the contacts of a case will be infected with constant probability $p_{t}$. The probability can be constant given two conditions: if 
the individual viral shedding is constant during a day, and if the contact duration is not considered for the calculation of the infection probability. The first condition is justifiable as viral shedding data is currently collected on a daily basis and there is no information available at a more granular time scale. The second condition is justifiable since the $\mathrm{AB}$ models are not used to test mitigation strategies aiming at reducing contact duration, and hence, this level of detail is not necessary.

In what follows, we extend Eq. 4 to account for the effect of different contact groups and asymptomatic infection on the probability of infection $p_{t}$.

Individuals in a community interact within their contact groups such as households, workplaces, schools, and other open places (e.g., stores and restaurants). To account for differences in the closeness of interactions in different contact groups, and the relative influence of these interactions on the infection probability, we consider that $\mathscr{N}_{t}=$ $\mathscr{N}_{t, h}+\mathscr{N}_{t, w}+\mathscr{N}_{t, o}$, where $h, w$, and $o$ are indices for household, work/school, and open places, respectively. We assume that $\mathscr{N}_{t, h} \sim \operatorname{bin}\left[c_{t, h}, p_{t, h}\right], \mathscr{N}_{t, w} \sim \operatorname{bin}\left[c_{t, w}, p_{t, w}\right]$, and $\mathscr{N}_{t, o} \sim \operatorname{bin}\left[c_{t, o}, p_{t, o}\right]$, where $c_{t, h}$ is the number of contact events that an infected case makes in the household between $t$ and $t+1$, and $p_{t, h}$ is the probability that an individual becomes infected after being contacted by an infected case in the household. Variables $c_{t, w}, p_{t, w}, c_{t, o}$, and $p_{t, o}$ can be defined similarly. Thus we can write from Eq. 3 that

$E\left[\mathscr{N}_{t, h}+\mathscr{N}_{t, w}+\mathscr{N}_{t, o}\right]=E[\mathscr{R}] \frac{E\left[\mathscr{V}_{t}\right]}{E\left[\sum_{t=0}^{\infty} \mathscr{V}_{t}\right]}$.

Using the binomial assumption, we can rewrite the above as

$c_{t, h} p_{t, h}+c_{t, w} p_{t, w}+c_{t, o} p_{t, o}=E[\mathscr{R}] \frac{E\left[\mathscr{V}_{t}\right]}{E\left[\sum_{t=0}^{\infty} \mathscr{V}_{t}\right]}$.

We assume that infection probability in the household is the highest and the probabilities in the workplaces/schools and open places are fractions of the household probability. Then we can write that $p_{t, w}=\kappa^{w} p_{t, h}$ and $p_{t, o}=\kappa^{o} p_{t, h}$, where $\kappa^{w}, \kappa^{o}<1.0$ and $\kappa^{w}>\kappa^{o}$. The above relationships can be argued from the fact that the duration and closeness of interactions at the households are highest followed by those for workplaces/schools and open places. We then have that

$p_{t, h}\left[c_{t, h}+\kappa^{w} c_{t, w}+\kappa^{o} c_{t, o}\right]=E[\mathscr{R}] \frac{E\left[\mathscr{V}_{t}\right]}{E\left[\sum_{t=0}^{\infty} \mathscr{V}_{t}\right]}$,

which yields

$p_{t, h}=\frac{E[\mathscr{R}] \frac{E\left[\mathscr{V}_{t}\right]}{E\left[\sum_{t=0}^{\left[\mathscr{V}_{t}\right]}\right.}}{c_{t, h}+\kappa^{w} c_{t, w}+\kappa^{o} c_{t, o}}$.

From the above, we get estimates of infection probabilities for an infected individual belonging to contact groups $h, w$, and $o$, during the time interval $t$ and $t+1$.
We can further extend the probability model to account for the fact that some infected cases could be asymptomatic. Presence of asymptomatic cases would naturally lower $E\left[\mathscr{N}_{t}\right]$, since their viral shedding is lower compared to the symptomatic cases [17]. We incorporate the probability of case being asymptomatic $\left(1-\pi_{S}\right)$ as follows:

$E[\mathscr{R}]=\pi_{s} * E\left[\mathscr{R}^{s}\right]+\left(1-\pi_{s}\right) * E\left[\mathscr{R}^{a}\right]$,

and

$E\left[\mathscr{R}^{a}\right]=\gamma * E\left[\mathscr{R}^{s}\right]$,

where $\gamma<1$ and $E\left[\mathscr{R}^{a}\right]$ and $E\left[\mathscr{R}^{s}\right]$ denote the reproduction numbers for asymptomatic and symptomatic cases, respectively. From Eqs. 6 and 7

$E\left[\mathscr{R}^{s}\right]=\frac{E[\mathscr{R}]}{(1-\gamma) \pi_{s}+\gamma}$.

Using Eqs. 7 and 8 we can extend the expression for $p_{t, h}$ in Eq. 5 for symptomatic and asymptomatic cases as follows:

$p_{t, h}^{s}=\frac{\frac{E[\mathscr{R}]}{(1-\gamma) \pi_{s}+\gamma} \frac{E\left[\mathscr{V}_{t}^{s}\right]}{E\left[\sum_{t=0}^{\infty} \mathscr{V}_{t}^{s}\right]}}{c_{t, h}+\kappa^{w} c_{t, w}+\kappa^{o} c_{t, o}}$,

and

$p_{t, h}^{a}=\frac{\gamma \frac{E[\mathscr{R}]}{(1-\gamma) \pi_{s}+\gamma} \frac{E\left[\mathscr{V}_{t}^{a}\right]}{E\left[\sum_{t=0}^{o} \mathscr{V}_{t}^{a}\right]}}{c_{t, h}+\kappa^{w} c_{t, w}+\kappa^{o} c_{t, o}}$.

The expressions for $p_{t, w}^{s}, p_{t, w}^{a}, p_{t, o}^{s}$, and $p_{t, o}^{a}$ can be derived and interpreted in a similar manner.

2.1 Obtaining values for the parameters of the epi model

The expressions in Eqs. 9 and 10 are designed to recreate the disease spread behavior among the agents of an AB model. We were particularly interested in an accurate reproduction of the spread during the initial stages of an outbreak (i.e., before the peak occurence), since this is the most uncertain period for policymaking.

In a nascent outbreak $E[\mathscr{R}]=E\left[\mathscr{R}^{0}\right]$, and there are two alternative inputs for this parameter. In the first alternative, the model user can select specific input values, which will be denoted as $R_{o}$. In the second alternative, the $E[\mathscr{R}]$ can be estimated from daily counts of new cases, as this is usually the only available information. The counts generally 
increase exponentially with rate $r$ during the inital phase of an outreak [56].

It is possible to find an estimate of the real reproduction number $\widetilde{R}$ in the initial phase of the infection using the Lotka-Euler equation [56]

$1=\widetilde{R} \sum_{t=0}^{\infty} \frac{E\left[\mathscr{V}_{t}^{s}\right]}{E\left[\sum_{t=0}^{\infty} \mathscr{V}_{t}^{s}\right]} e^{-r t}$,

where $\widetilde{R}$ is an adjusted value of $E\left[\mathscr{R}^{0}\right]$, since it accounts for the fact that some of the real population have partial immunity to influenza viruses. Hence, use of $\widetilde{R}$ for $E\left[\mathscr{R}^{0}\right]$, incorporates population immunity to the model. Note that, if Eq. 11 is used to obtain $E\left[\mathscr{R}^{0}\right]$ for the $\mathrm{AB}$ model, both Eq. 11 and the model should assume the same $\frac{E\left[\mathscr{W}_{t}^{s}\right]}{E\left[\sum_{t=0}^{\infty} \mathscr{Y}_{t}^{s}\right]}$.

With regards to the other parameters in expressions Eqs. 9 and 10, values for $\frac{E\left[\mathscr{V}_{t}^{s}\right]}{E\left[\sum_{t=0}^{\infty} \mathscr{Y}_{t}^{s}\right]}$ and $\frac{E\left[\mathscr{V}_{t}^{a}\right]}{E\left[\sum_{t=0}^{\infty} \mathscr{Y}_{t}^{a}\right]}$ can be derived by using readily available information about viral shedding profiles, and the relationship between symptomatic and asymptomatic viral shedding [17]. Values for $\gamma, \pi_{s}, \kappa^{w}$ and $\kappa^{o}$ are obtained from the literature [17, 41]. The parameters $c_{t, h}, c_{t, w}$ and $c_{t, o}$ are obtained from the $\mathrm{AB}$ model.

If the daily counts of new cases are underreported or overreported, these data should be adjusted accordingly. Section 4.2 describes an implementation example, including a situation of case underreporting.

\subsection{Modeling co-circulating pandemic and seasonal influenza viruses}

We now extend our model to account for two co-circulating influenza viruses: pandemic (PI), and seasonal (SI). The PI virus has a high transmission potential given by a high value of the expected reproduction number $E\left[\mathscr{R}_{P I}\right]$. Conversely, The SI virus has a low transmission potential given by a low value for the $E\left[\mathscr{R}_{S I}\right]$. The infection probabilities for the two circulating viruses can then be given from Eq. 4 as follows.

$p_{t, P I}=\frac{E\left[\mathscr{R}_{P I}\right] \frac{E\left[\mathscr{\mathscr { V }}_{t, P I}\right]}{E\left[\sum_{t=0}^{\infty} \mathscr{\mathscr { V }}_{t, P I}\right]}}{c_{t}}$,

and

$$
p_{t, S I}=\frac{E\left[\mathscr{R}_{S I}\right] \frac{E\left[\mathscr{\mathscr { V }}_{t, S I}\right]}{E\left[\sum_{t=0}^{\infty} \mathscr{\mathscr { N }}_{t, S I}\right]}}{c_{t}} .
$$

Considering various contact groups and asymptomatic infection, the infection probabilities can be given from Eqs. 9 and 10 as

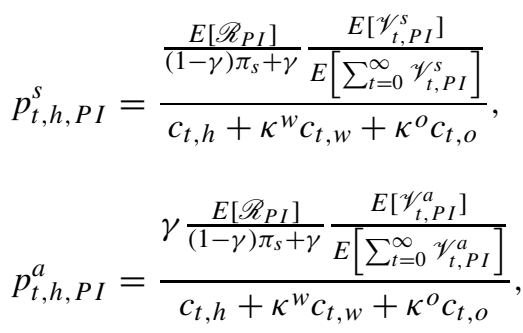

$p_{t, h, S I}^{s}=\frac{\frac{E\left[\mathscr{R}_{S I}\right]}{(1-\gamma) \pi_{s}+\gamma} \frac{E\left[\mathscr{\mathscr { V }}_{t, S I}^{s}\right]}{E\left[\sum_{t=0}^{\infty} \mathscr{Y}_{t, S I}^{s}\right]}}{c_{t, h}+\kappa^{w} c_{t, w}+\kappa^{o} c_{t, o}}$,

$p_{t, h, S I}^{a}=\frac{\gamma \frac{E\left[\mathscr{R}_{S I}\right]}{(1-\gamma) \pi_{s}+\gamma} \frac{E\left[\mathscr{\mathscr { V }}_{t, S I}^{a}\right]}{E\left[\sum_{t=0}^{\infty} \mathscr{Y}_{t, S I}^{a}\right]}}{c_{t, h}+\kappa^{w} c_{t, w}+\kappa^{o} c_{t, o}}$.

Equations for $p_{t, w, P I}^{s}, p_{t, w, P I}^{a}, p_{t, w, S I}^{s}, p_{t, w, S I}^{a}, p_{t, o, P I}^{s}$, $p_{t, o, P I}^{a}, p_{t, o, S I}^{s}$, and $p_{t, o, S I}^{a}$ can be derived similarly. Note that the parameters $\gamma$ and $\pi_{s}$ could also be considered to have separate values for PI and SI.

When a pandemic influenza virus co-circulates with one of its seasonal antigenic variants, the disease progression in each individual is determined by the epidemiological features of an infectious contact event. These features are the following:

- The strains in a contacting (infected) person: a contacting person can be infected with a pandemic influenza (PI) strain, a seasonal influenza (SI) strain, or both (PI, SI).

- The strains transmitted by a contacting person: the strains transmitted could be PI, SI or both.

- The infection status of a contacted person: a contact is only a candidate for future infection in the following ways: if the contact has not been infected with any viral strain (no infection); the contact has already recovered from an infection with either PI or SI; the contact is already infected with either PI (ongoing PI) or SI (ongoing SI).

A combination of features in the contact event results in an infection state in the contacted person. The possible resulting infection states are: PI infection, SI infection, recovery from SI and infection with PI (re-infection PI), recovery from PI and infection with SI (re-infection SI), ongoing infection with SI and new infection with PI (coinfection PI), ongoing infection with PI and new infection with SI (co-infection SI), and simultaneous infection with PI and SI (simultaneous).

Table 1 shows all the possibilities for a contact event that might result in the infection of a contacted person. For 
Table 1 Possible contact events resulting in an infection of a contacted individual

\begin{tabular}{|c|c|c|c|c|}
\hline $\begin{array}{l}\text { Strains in contacting } \\
\text { (infected) person }\end{array}$ & Strains transmitted & $\begin{array}{l}\text { Previous infection status } \\
\text { of contacted person }\end{array}$ & $\begin{array}{l}\text { Resulting infection status } \\
\text { of contacted person }\end{array}$ & Cross-immunity factor \\
\hline \multirow[t]{3}{*}{ PI } & \multirow[t]{3}{*}{ PI } & No infection & Infection PI & None \\
\hline & & Recovered from SI & Re-infection PI & $\varepsilon_{S I-P I}^{r}$ \\
\hline & & Ongoing SI & Co-infection PI & $\varepsilon_{S I-P I}^{c}$ \\
\hline \multirow[t]{3}{*}{ SI } & \multirow[t]{3}{*}{ SI } & No infection & Infection SI & None \\
\hline & & Recovered from PI & Re-infection SI & $\varepsilon_{P I-S I}^{r}$ \\
\hline & & Ongoing PI & Co-infection SI & $\varepsilon_{P I-S I}^{c}$ \\
\hline \multirow[t]{3}{*}{ PI, SI } & \multirow[t]{3}{*}{ PI } & No infection & Infection PI & None \\
\hline & & Recovered from SI & Re-infection PI & $\varepsilon_{S I-P I}^{r}$ \\
\hline & & Ongoing SI & Co-infection PI & $\varepsilon_{S I-P I}^{c}$ \\
\hline \multirow[t]{3}{*}{ PI, SI } & \multirow[t]{3}{*}{ SI } & No infection & Infection SI & None \\
\hline & & Recovered from PI & Re-infection SI & $\varepsilon_{P I-S I}^{r}$ \\
\hline & & Ongoing PI & Co-infection SI & $\varepsilon_{P I-S I}^{c}$ \\
\hline \multirow[t]{6}{*}{ PI, SI } & \multirow[t]{6}{*}{ PI, SI } & No infection & Simultaneous & $\varepsilon_{S I / P I}^{s}$ \\
\hline & & & & $\varepsilon_{P I / S I}^{s}$ \\
\hline & & Recovered from SI & Re-infection PI & $\varepsilon_{S I-P I}^{r}$ \\
\hline & & Ongoing SI & Co-infection PI & $\varepsilon_{S I-P I}^{c}$ \\
\hline & & Recovered from PI & Re-infection SI & $\varepsilon_{P I-S I}^{r}$ \\
\hline & & Ongoing PI & Co-infection SI & $\varepsilon_{P I-S I}^{c}$ \\
\hline
\end{tabular}

example, the first possibility shown in Table 1 indicates that the contacting person is only infected with PI and effectively transmits PI to a contact with three possible previous infection conditions (column 3, row 1). The possible infection status of the contacted person is shown in column 4 . We are assuming in the co-circulation model that if a contacted person is co-infected, re-infected, or recovered from both PI and SI, she will not be infected from any of the two viruses anymore. We also assume that co-infection will not generate a new influenza virus subtype. Column 5 shows the notation for cross-immunity factors considered in the epi model for co-circulating PI and SI. Cross-immunity might occur once one of the viruses generates an immune response that reduces the spread of the other co-circulating virus.

Once an individual $i$ is in one of the above co-infection, re-infection or simultaneous infection states, we assume that the individual's viral shedding profiles are reduced by the cross-immunity factors $\varepsilon_{j}^{l}$, depending the virus generating the cross-immunity. The index $j$ is denoted $S I / P I$ if the individual was simultaneously infected and PI generates the cross-immunity; $P I / S I$ if the individual was simultaneously infected and SI generates the crossimmunity, $S I-P I$, if the individual was infected with SI first and then infected with PI, and $P I-S I$, for the reverse sequence. The index $l \in r, c, s$ indicates the presence of re-infection $(r)$, co-infection $(c)$ or simultaneous infection $(s)$. The $\varepsilon_{j}^{l}$ accounts for the cell-mediated and antibody-mediated cross-protection within the human body [20, 31, 54]. Note that, though we have considered crossimmunity in our $\mathrm{AB}$ model, to our knowledge, realistic numerical values for the $\varepsilon_{j}^{l}$ for all $j$ and $l$ combinations are not available in the open literature. Per the literature cited above, research on immunology appears to be in the early stages of addressing this issue. In the numerical implementation of our calibration methodology, we assumed a value of one for all $\varepsilon_{j}^{l}$, indicating no crossimmunity. In the Appendix A, we test and discuss the effect of varying the cross-immunity factors in the epi model.

\section{Implementation of the epi model within an AB simulation}

The epi model is designed to be embedded in an AB simulation model. The simulation emulates the daily interactions among the individuals of the population of an outbreak region, and the spread of the influenza viruses among the population. Figure 1 shows a schematic of the AB simulation used as the testbed for the epi model. Block $8 \mathrm{~b}$ of Fig. 1 shows the placement of the epi model in the simulation. The number of contacts, the time since infection, and the symptomatic status per infected case are inserted 


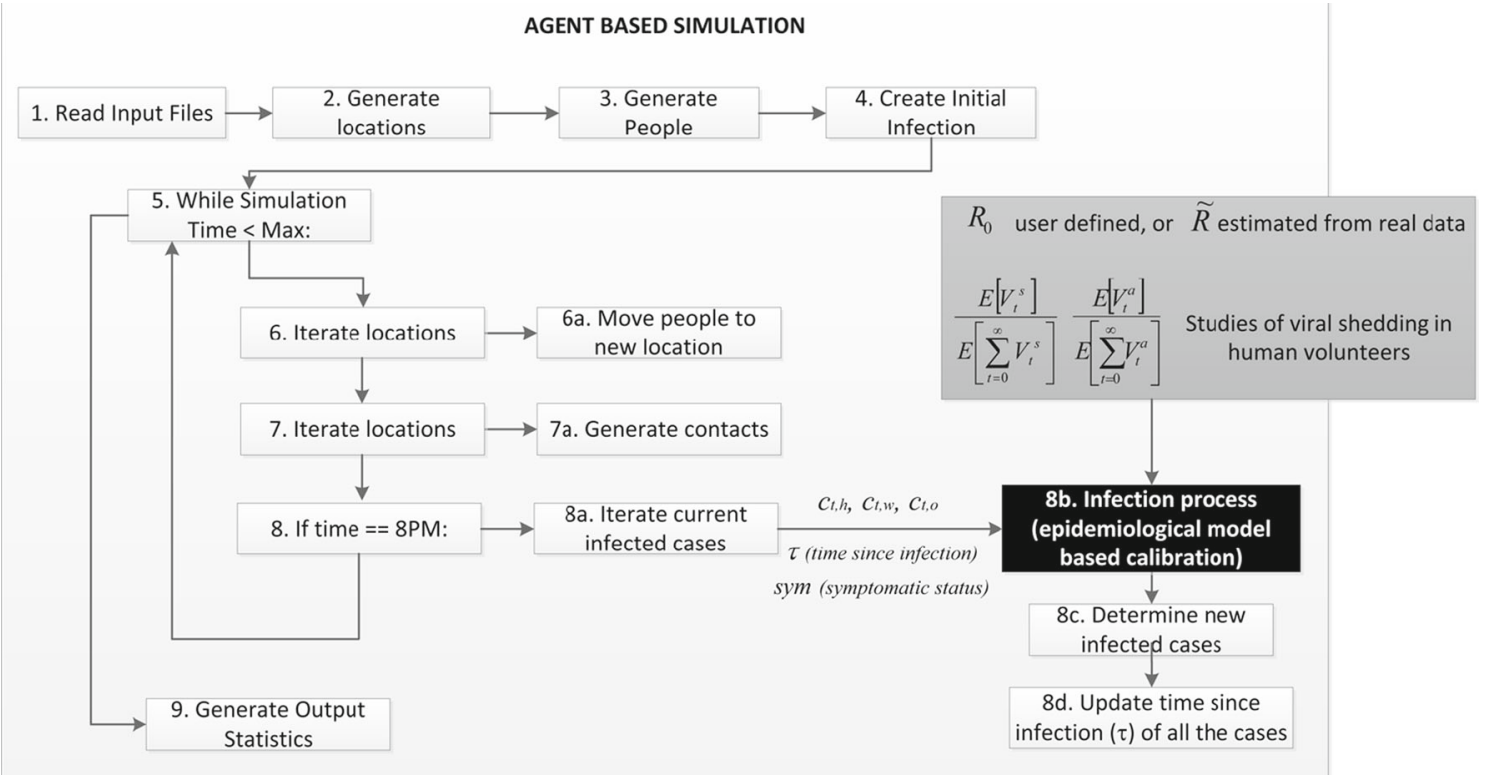

Fig. 1 a Schematic of the AB simulation used as testbed for the epidemiological model

directly from the simulation to the epidemiological model, while the reproduction numbers and the viral shedding profile are obtained from external sources, as described in the Fig. 1.

The implementation of the epi model is summarized in the algorithm 1.

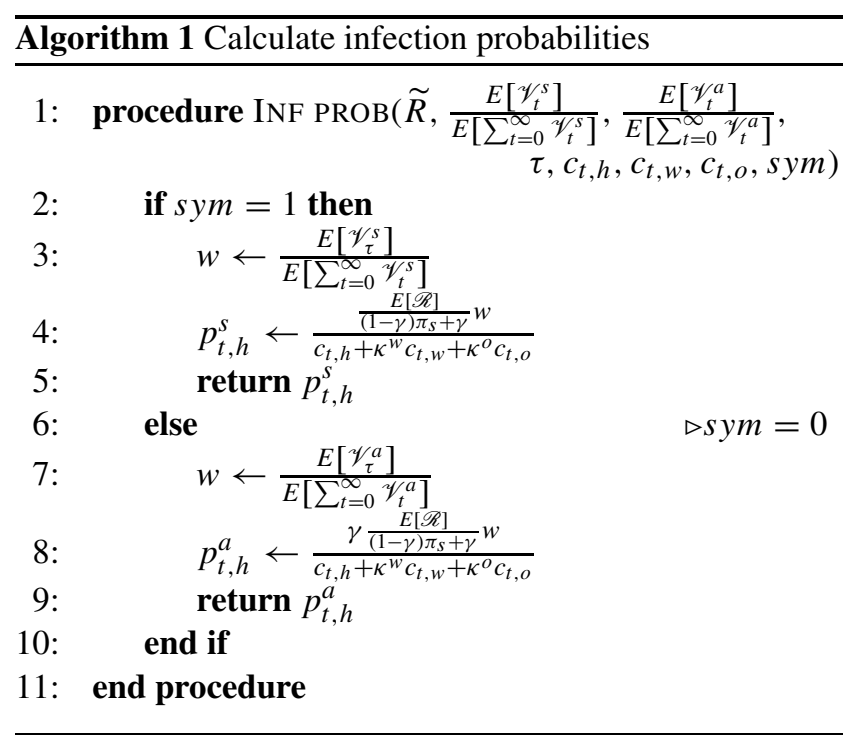

\subsection{Complexity of the implemented epi model}

Existing $\mathrm{AB}$ models have translated epidemiological data/ parameters to the internal model parameters with humanassisted and algorithmic-driven calibration. In the humanassisted calibration, the user manually fits the internal parameters to the external information, which in most of the cases is the disease transmission paramater $R_{o}[9,15,29$, $30,32,36,37,53,55]$. Algorithmic-driven calibration has been developed by using nonlinear optimization to simultaneously fit several external parameters, including $R_{o}$ and $\pi^{s}$ [58], or by using stochastic search to fit the real cumulative number of ILI cases [44].

In this section we demonstrate how the assumptions in an epi model dictate the complexity of the calibration of the epi parameters. In our demonstration, we compare the complexity and assumptions of an existing epi model with those of our epi model. The existing epi model is embedded into an AB simulation called Epifast [44]. Epifast uses a simulation based optimization approach to fit the core parameters of their epi model to Flutrends ILI data. They define a parameter $X_{t}$ as the "probability of transmission per unit of contact time given contact between an infectious and a susceptible individual." This parameter is housed within the equation of the overall probability of transmission between susceptible $u$ and infectious $v$ as follows:

$p(w(u, v))=1-\left(1-X_{t}\right)^{w(u, v)}$,

where $w(u, v)$ is the contact duration. To fit $X_{t}$ to the Flutrends data, stochastic search through the RobbinsMonro algorithm is used. Define $\alpha_{t}$ as the real number of ILI counts at $t$ (from Flutrends), $X_{t, n}$ as the disease transmissibility at iteration step $n$ and $t$, and $M_{t}\left(X_{t, n}\right)$ as the simulated cumulative number of ILI counts. The the Robbins-Monro algorithm can be described as in Algorithm 2. 


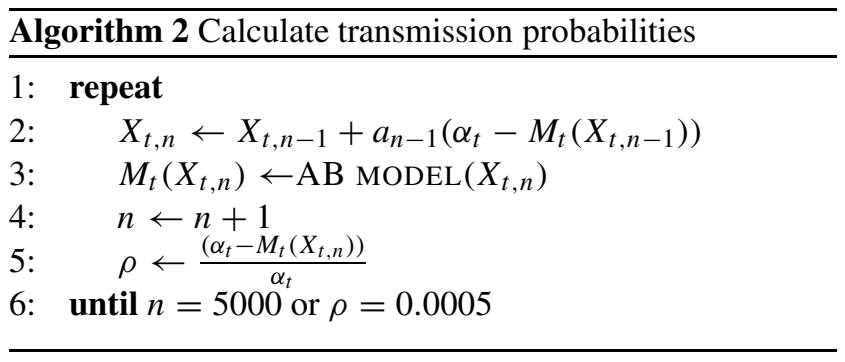

Where $a_{n-1}$ is a positive value that satisfies the conditions described in [44]. In algorithm 2, the procedure $A B$ MODEL is called in step 3, once a new $X_{t, n}$ is selected. Algorithm 2 reaches convergence when the error rate, $\rho$ is 0.0005 . If $n=5000$ and convergence has not been achieved, then the stochactic search procedure is invoked as many times as needed, until convergence.

To compare algorithm 1 and 2, we define a basic operation as an assignment (e.g., $a \leftarrow b$ ). In algorithm 1, for each infected case at time t, there are 2 operations needed to compute the infection probability, and the algorithm complexity is $O$ (1) (constant). For algorithm 2, we first disregard the complexity embedded in step 3 . In the best case (i.e., finding convergence in the first iteration), the complexity is $O(1)$. However, if step 3 is considered, the complexity will be higher that $O(1)$ since Epifast executes several loops [13]. This comparison helps to illustrate that any iterative fitting procedure will have a best case complexity of $O(1)$, which will only occur if convergence is reached in the first iteration. Otherwise, the complexity will increase with the number of iterations, at the very least. In contrast, our complexity is basic and constant. Note, however, that the calibration of Epifast currently requires an iterative procedure, since the model in Eq. 18 assumes contact duration, and the computation of the infection probability will take several time steps in the simulation.

Our selection of Epifast for the purpose of comparison was due to a number of reasons. Epifast uses state of the art in calibration methods for real time data. In addition, their results are reported by fitting one parameter, and the fitting algorithm is completely reported, which facilitates our discussion on their complexity. Finally Epifast is very well described in the literature [13].

\subsection{Daily interactions of people (agents) in an urban area}

At the beginning of the simulation, each individual is created and assigned to an age group, a household, a school/workplace, and a set of errand places. Individuals are set to visit these locations by following a daily hour by hour schedule, as in [21]. The schedule assignments are probabilistic, and were calculated based on data for the
Hillsborough county in Tampa, Florida, USA. The probability distributions were reported in [55], and were obtained from the 2002 U.S. Economic Census, the 2001 American Community Survey, and the 2001 National Household Travel Survey. These processes are summarized in steps 1 , 2, and 3 of Fig. 1.

The simulation runs hour by hour for each day. Each individual in each hour is placed in a location (mixing group) following his/her daily schedule. Once they are in a mixing group, individuals randomly make a number of contacts (Fig. 1, steps 5, 6, and 7). The number of contacts in different mixing groups were adopted from a recent survey on daily contact patterns in eight European countries [41]. To our knowledge, no such study was conducted for the U.S. Hence, we consider the European social behavior as an approximation for the mixing behavior in the U.S. Table 2, (columns 2 and 3) shows the daily number of contacts and proportion of contacts occurring in the mixing groups/locations [41]. Column 4 of the table shows the number of contacts that each individual makes per location setting, which is obtained by multiplying the numbers in columns 2 and 3.

\subsection{Spread of the influenza viruses}

To begin an outbreak, a set of randomly selected individuals (cases) are infected. Each infected case $i$ contacts other individuals as described in Section 3.2. The contacts of $i$ are aggregated to compute the value of $c_{t}=c_{t, h}+c_{t, w}+c_{t, o}$, for day $t$. At the end of day $t$, new infected cases appear from the individuals contacted by each of the previously infected

Table 2 Number of contacts an individual makes per day and per location setting

\begin{tabular}{llll}
\hline $\begin{array}{l}\text { Location type } \\
\text { (mixing group) }\end{array}$ & $\begin{array}{l}\text { Number } \\
\text { of contacts } \\
\text { per day }\end{array}$ & $\begin{array}{l}\text { Proportion } \\
\text { of contacts } \\
\text { occurring in } \\
\text { the location }\end{array}$ & $\begin{array}{l}\text { Number of } \\
\text { contacts in } \\
\text { the location } \\
\text { per day }\end{array}$ \\
\hline Home & 13.4 & 0.23 & 3 \\
Factory & 14 & 0.21 & 3 \\
Office & 14 & 0.21 & 3 \\
Pre-school & 10.21 & 0.14 & 2 \\
Elementary & 14.81 & 0.14 & 2 \\
Middle school & 18.22 & 0.14 & 3 \\
High school & 17.6 & 0.14 & 3 \\
University & 15.98 & 0.14 & 2 \\
Afterschool & 13.4 & 0.14 & 2 \\
Grocery & 13.4 & 0.16 & 2 \\
Restaurant & 13.4 & 0.16 & 2 \\
Entertainment & 13.4 & 0.16 & 2 \\
Church & 13.4 & 0.16 & 2 \\
\hline
\end{tabular}


cases based on the epi model in Section 2 (Fig. 1, step 8). This process repeats at every new day, thus spreading the virus(es). The $\mathrm{AB}$ model does not assume alterations of the contact patterns as a behavioral response to the disease spread (e.g., work absenteeism).

Table 3 shows the viral shedding data that we have used here. We used data corresponding to the $\mathrm{A} / \mathrm{H} 1 \mathrm{~N} 1$ virus, as presented in the literature (which aggregates the viral shedding profiles per virus type, and subtype). The viral titers in asymptomatic individuals, as reported in column 4 , were obtained by assuming that the viral titer from symptomatic cases who shed virus and develop illness is $2 \log _{10}$ times higher than individuals who do not develop symptoms [17].

Since the seasonal H1N1 is an antigenic variant of the pandemic $\mathrm{H} 1 \mathrm{~N} 1$, we assumed the same viral shedding profile for the symptomatic cases of H1N1 PI and SI, that is

$$
\begin{aligned}
& \frac{E\left[\mathscr{V}_{t, P I}\right]}{E\left[\sum_{t=0}^{\infty} \mathscr{V}_{t, P I}\right]}=\frac{E\left[\mathscr{V}_{t, S I}\right]}{E\left[\sum_{t=0}^{\infty} \mathscr{V}_{t, S I}\right]}=\frac{E\left[\mathscr{V}_{t, P I}^{s}\right]}{E\left[\sum_{t=0}^{\infty} \mathscr{V}_{t, P I}^{s}\right]} \\
& =\frac{E\left[\mathscr{V}_{t, S I}^{s}\right]}{E\left[\sum_{t=0}^{\infty} \mathscr{V}_{t, S I}^{s}\right]} .
\end{aligned}
$$

We also assumed the same viral shedding profile for the asymptomatic cases of H1N1 PI and SI, that is

$$
\frac{E\left[\mathscr{V}_{t, P I}^{a}\right]}{E\left[\sum_{t=0}^{\infty} \mathscr{V}_{t, P I}^{a}\right]}=\frac{E\left[\mathscr{V}_{t, S I}^{a}\right]}{E\left[\sum_{t=0}^{\infty} \mathscr{V}_{t, S I}^{a}\right]}
$$

Table 4 shows the other parameter values that were used to calculate the infection probabilities.

Table 3 Daily viral titer count in symptomatic and asymptomatic individuals inoculated with influenza A/H1N1 [17]

\begin{tabular}{llll}
\hline Days & Hours & $\begin{array}{l}\text { Viral titer in a } \\
\text { symptomatic } \\
\text { individual } \\
\text { (log scale) }\end{array}$ & $\begin{array}{l}\text { Viral titer in an } \\
\text { asymptomatic } \\
\text { individual } \\
\text { (log scale) }\end{array}$ \\
\hline $0-1$ & $0-24$ & 0.1 & 0.05 \\
$1-2$ & $24-48$ & 1.75 & 0.875 \\
$2-3$ & $48-72$ & 3 & 1.5 \\
$3-4$ & $72-96$ & 2.5 & 1.25 \\
$4-5$ & $96-120$ & 1.8 & 0.9 \\
$5-6$ & $120-144$ & 1.2 & 0.6 \\
$6-7$ & $144-168$ & 0.7 & 0.35 \\
$7-8$ & $168-192$ & 0.5 & 0.25 \\
$8-9$ & $192-216$ & 0.2 & 0.1 \\
\hline
\end{tabular}

Table 4 Parameter values used to calculate the infection probabilities

\begin{tabular}{lll}
\hline Parameter & Value & Reference \\
\hline$E\left[R_{P I}^{0}\right]$ & $1.6-3.0$ & {$[23,24,27,57]$} \\
$E\left[R_{S I}^{0}\right]$ & 1.3 & {$[18]$} \\
$\gamma$ & 0.22 & {$[17]$} \\
$\pi_{S}$ & 0.669 & {$[17]$} \\
$\kappa^{w}$ & 0.67 & {$[41]$} \\
$\kappa^{o}$ & 0.44 & {$[41]$} \\
\hline
\end{tabular}

\section{Results}

Our model accepts a field estimate of $R_{o}$, and then uses it to update (calibrate) the infection probabilities in a way that the combined effect of the given virus epidemiology, demographics, and social behavior results in an infection trend yielding a similar value of $\widehat{R}_{o}$. In the AB model, disease spread occurs probabilistically, and therefore the epi model should be tested for calibration accuracy. The accuracy is measured by the closeness of the output value $\widehat{R}_{O}$ to the input value $R_{o}$. The accuracy will be observed in three cases: 1) when we assume user defined values of the $R_{O}$, and we calculate $\widehat{R}_{O}$ by using the direct definition of the basic reproduction number, 2) when both $R_{o}$ and $\widehat{R}_{o}$ are calculated using the trend of real and simulated incidence data, respectively, and 3) when the epidemiological model includes an additional outbreak, symptomatic cases, and differences in the contact groups.

4.1 Accuracy of the epi model with user defined $R_{O}$

In this case, the infected individuals from the contacts are given by Eq. 12, where only PI is in effect, and not considered are asymptomatic cases and differences in the contact groups.

A user defined $R_{0}$ is introduced in Eq. 12 by equating it to the term $E\left[\mathscr{R}_{P I}\right]$ of the individuals in $k=0$. For a population of around 100,000 inhabitants and 10 cases in generation $k=0$, Table 5 shows the average $\widehat{R}_{o}$ obtained when different values for $R_{O}$ are introduced in the simulation.

The reproduction number $\widehat{R}_{o}$ was calculated by the ratio between the number of infected cases in a generation $k+1$ and the number of infected cases in generation $k$. Though theoretically a maximum value of $\widehat{R}_{o}$ should be observed at $k=0$, in simulated experiments peak values of $\widehat{R}_{o}$ were often obtained at higher values of $k$ (up to $k=5$ ). For an $R_{o}=2.0$ (first part of Table 5), an average $\widehat{R}_{o}$ of 2.083 was obtained over 143 replicates, with $(1.997,2.168)$ as the $95 \%$ confidence interval (CI). For 48 out of 143 replicates, 
Table 5 Values for the $\widehat{R}_{0}$ obtained for an outbreak region with 100,000 inhabitants

\begin{tabular}{|c|c|c|c|c|c|c|c|}
\hline$R_{0}=2.0$ & $k=0$ & $k=1$ & $k=2$ & $k=3$ & $k=4$ & $k=5$ & \\
\hline Replicates that peaked on $k$ & 48 & 35 & 24 & 17 & 11 & 8 & 143 (total) \\
\hline Average $\widehat{R}_{o}$ over replicates & 2.173 & 2.079 & 2.072 & 2.002 & 1.941 & 1.961 & 2.083 \\
\hline Lower $95 \%$ CI & 2.087 & 2.011 & 1.965 & 1.936 & 1.846 & 1.835 & 1.997 \\
\hline Upper $95 \%$ CI & 2.256 & 2.146 & 2.179 & 2.069 & 2.036 & 2.087 & 2.168 \\
\hline$R_{0}=2.5$ & $k=0$ & $k=1$ & $k=2$ & $k=3$ & $k=4$ & $k=5$ & \\
\hline Replicates that peaked on $k$ & 48 & 35 & 18 & 16 & 11 & 7 & 135 (total) \\
\hline Average $\widehat{R}_{o}$ over replicates & 2.740 & 2.502 & 2.373 & 2.372 & 2.317 & 2.337 & 2.53 \\
\hline Lower $95 \%$ CI & 2.632 & 2.438 & 2.295 & 2.322 & 2.257 & 2.281 & 2.451 \\
\hline Upper $95 \%$ CI & 2.847 & 2.567 & 2.452 & 2.423 & 2.377 & 2.392 & 2.610 \\
\hline$R_{0}=3.0$ & $k=0$ & $k=1$ & $k=2$ & $k=3$ & $k=4$ & $k=5$ & \\
\hline Replicates that peaked on $k$ & 49 & 31 & 15 & 19 & 19 & 17 & 150 (total) \\
\hline Average $\widehat{R}_{o}$ over replicates & 3.150 & 2.932 & 2.858 & 2.792 & 2.713 & 2.668 & 2.920 \\
\hline Lower $95 \%$ CI & 3.062 & 2.878 & 2.738 & 2.741 & 2.673 & 2.639 & 2.854 \\
\hline Upper $95 \%$ CI & 3.236 & 2.987 & 2.978 & 2.842 & 2.753 & 2.698 & 2.987 \\
\hline
\end{tabular}

peak $\widehat{R}_{o}$ were observed at $k=0$. The number of replicates for which peak $\widehat{R}_{o}$ values were observed at $k=1,2,3,4$, and 5 are $35,24,17,11$, and 8 , respectively.

For an $R_{O}=2.5$ (second part of Table 5), an average of 135 replicates provides $\widehat{R}_{o}=2.53$, with CI $(2.451,2.610)$. For an $R_{o}=3.0$, an average of 150 replicates provides $\widehat{R}_{o}=2.920$, with CI $(2.854,2.987)$. For a larger population of around 1,000,000 inhabitants and 10 cases in generation zero, Table 6 presents the results for the average $\widehat{R}_{o}$ when $R_{O}=2.0, R_{o}=2.5$, and $R_{o}=3.0$ are introduced in the simulation.
These results indicate that our model obtains infection probabilities that yield an average $\widehat{R}_{O}$ similar to the basic reproduction number $R_{O}$ introduced to the simulation. The calibration approach produce robust values for $\widehat{R}_{o}$, since the CIs are small and in all but one scenarios, the CIs include the target values. In the scenario $\left(R_{o}=3\right.$, Table 5$)$, the target $R_{o}$ is not included in the $\mathrm{CI}$, and it is 0.013 units away from the upper CI. This deviation can be considered insignificant, since sensitivity analyses of $R_{o}$ in the literature are typically performed at a minimum step size of 0.2 units [24, 27, 33, $38,51]$.

Table 6 Values for the $\widehat{R}_{o}$ obtained for an outbreak region with 1,000,000 inhabitants

\begin{tabular}{|c|c|c|c|c|c|c|c|}
\hline$R_{0}=2.0$ & $k=0$ & $k=1$ & $k=2$ & $k=3$ & $k=4$ & $k=5$ & \\
\hline Replicates that peaked on $k$ & 41 & 32 & 18 & 24 & 12 & 8 & 135 (total) \\
\hline Average $\widehat{R}_{O}$ over replicates & 2.229 & 2.163 & 2.112 & 1.986 & 1.928 & 1.949 & 2.111 \\
\hline Lower $95 \%$ CI & 2.150 & 2.073 & 1.980 & 1.935 & 1.852 & 1.835 & 2.026 \\
\hline Upper $95 \%$ CI & 2.309 & 2.252 & 2.243 & 2.038 & 2.003 & 2.064 & 2.197 \\
\hline$R_{0}=2.5$ & $k=0$ & $k=1$ & $k=2$ & $k=3$ & $k=4$ & $k=5$ & \\
\hline Replicates that peaked on $k$ & 46 & 37 & 24 & 14 & 10 & 6 & 137 (total) \\
\hline Average $\widehat{R}_{O}$ over replicates & 2.641 & 2.584 & 2.476 & 2.362 & 2.343 & 2.345 & 2.533 \\
\hline Lower $95 \%$ CI & 2.569 & 2.508 & 2.414 & 2.299 & 2.258 & 2.275 & 2.462 \\
\hline Upper $95 \%$ CI & 2.713 & 2.660 & 2.537 & 2.425 & 2.428 & 2.415 & 2.605 \\
\hline$R_{0}=3.0$ & $k=0$ & $k=1$ & $k=2$ & $k=3$ & $k=4$ & $k=5$ & \\
\hline Replicates that peaked on $k$ & 43 & 29 & 19 & 10 & 6 & 7 & 114 (total) \\
\hline Average $\widehat{R}_{o}$ over replicates & 3.128 & 2.948 & 2.822 & 2.736 & 2.719 & 2.720 & 2.95 \\
\hline Lower $95 \%$ CI & 3.039 & 2.861 & 2.780 & 2.675 & 2.668 & 2.637 & 2.874 \\
\hline Upper $95 \%$ CI & 3.217 & 3.036 & 2.865 & 2.796 & 2.770 & 2.803 & 3.026 \\
\hline
\end{tabular}


The model performance holds for different population levels. The slight variations between the input value of $R_{O}$ and the observed average value of $\widehat{R}_{O}$ can be attributed to the stochastic disease spread process embedded in the simulation and possible instabilities that simulation models normally present at their initial stages.

We further explored the effect of the number of replicates and the initial number of infected cases on the accuracy of the calibration approach. Figure 2a shows the decreasing width of the CIs as the number of replicates are increased. With 30 initial infected cases, accuracy is achieved for $R_{O}=$ $2, R_{o}=2.5$ and $R_{o}=3.0$. For $R_{o}=1.5$, accuracy is not achieved as the $\widehat{R}_{o}=1.58$ for 100 replicates. Figure $2 \mathrm{~b}$ shows that the value of $R_{O}=1.5$ falls within the confidence interval obtained for $\widehat{R}_{O}$ as the initial number of infected cases is increased to 120 . We conclude that, for small values of the infection probabilities, small number of initial infected cases might affect the accuracy of the approach. Figure $2 \mathrm{~b}$ also shows the unstable behavior of $\widehat{R}_{o}=3.0$ as the number of initial infected cases increase. We believe this case is showing the effect of infection probabilities that are obtained by using $R_{O}>=3.0$, in combination with small number of candidate contacs for infection (e.g., a infected case having 1 candidate contact for infection during a day). Such situation can create undefined values for the infection probabilities. As we did not observe any unusual pattern with 30 initial infected cases, we did not discard $R_{o}=3.0$ as a possible input, but we discard the use of the calibration approach for any values higher than $R_{o}=3.0$.

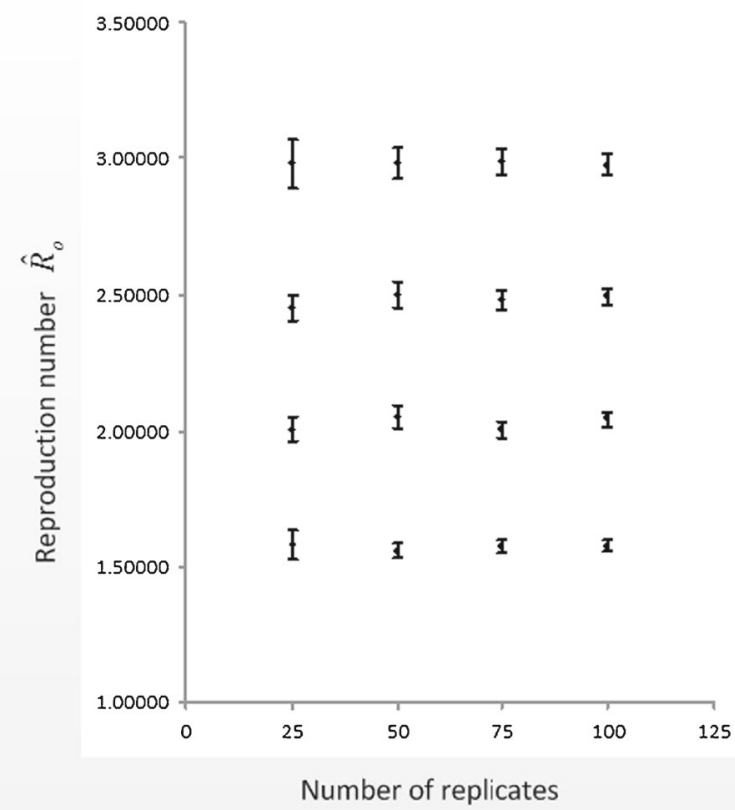

Fig. 2 a CI on reproduction numbers $\widehat{R}_{o}$ obtained for different number of replicates. For all the replicates the initial number of infected cases was randomly chosen and set to 30 individuals. b CI on
Additional 30 replicates were run to investigate the role of the infected cases if they are not randomly selected, but they are chosen from a specific age group and location. For an $R_{o}>=1.8$, and selecting 30 adults from the same workplace, The CI obtained for $\widehat{R}_{o}$ is $(1.65,1.84)$. If 30 children are selected from the same school, the CI obtained is (1.67, 1.82). A non-significant reduction was observed when children are the initial infected cases, which we attribute to some cases trying to re-infect susceptibles in their contact network with the same virus, which is not possible in our model.

The replicates used in the results were run using OpenMP with 4 simulations running in parallel in 2 cores. For a population of 1.000 .000 and 100 days, it took 8 min to run 4 replicates simultaneously.

\subsection{Estimating parameters for the epi model from real outbreak data}

Using real data on the daily number of specimens submitted and confirmed with PI in the Florida region, we estimated exponential growth rates for the initial phase of the outbreak, which were used in Eq. 11 to find the reproduction number $\widetilde{R}$.

Figure $3 \mathrm{a}$ shows the raw data obtained from the Florida Bureau of Laboratories (BOL). The discontinuities observed correspond to the Sundays where no submissions or confirmations were recorded. To complete the series, we fitted a poisson curve to the data, which in turn assumes that

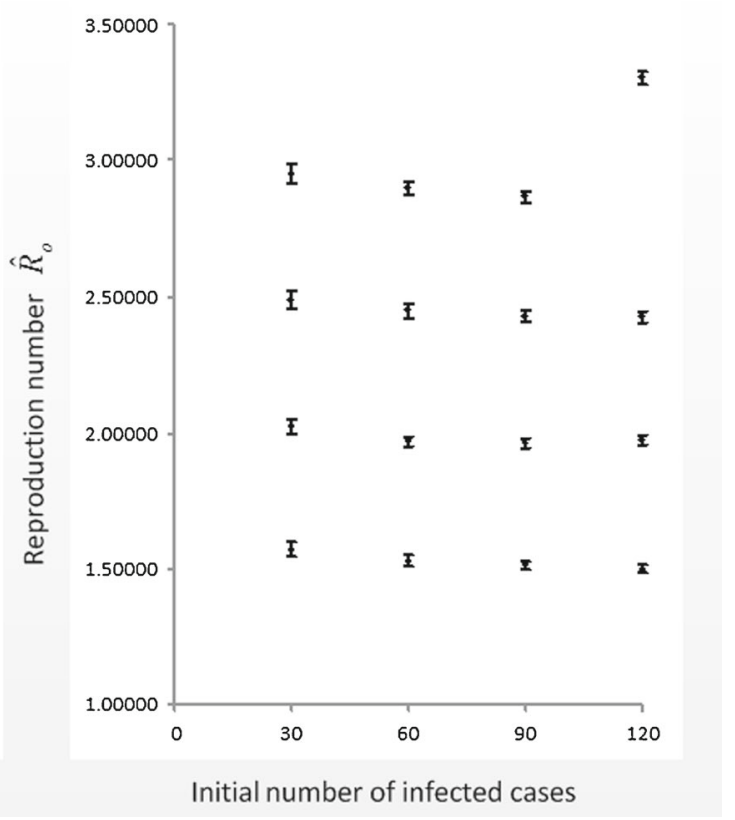

reproduction numbers $\widehat{R}_{O}$ obtained for different numbers of initial infected cases. The confidence intervals were obtained using 100 replicates 


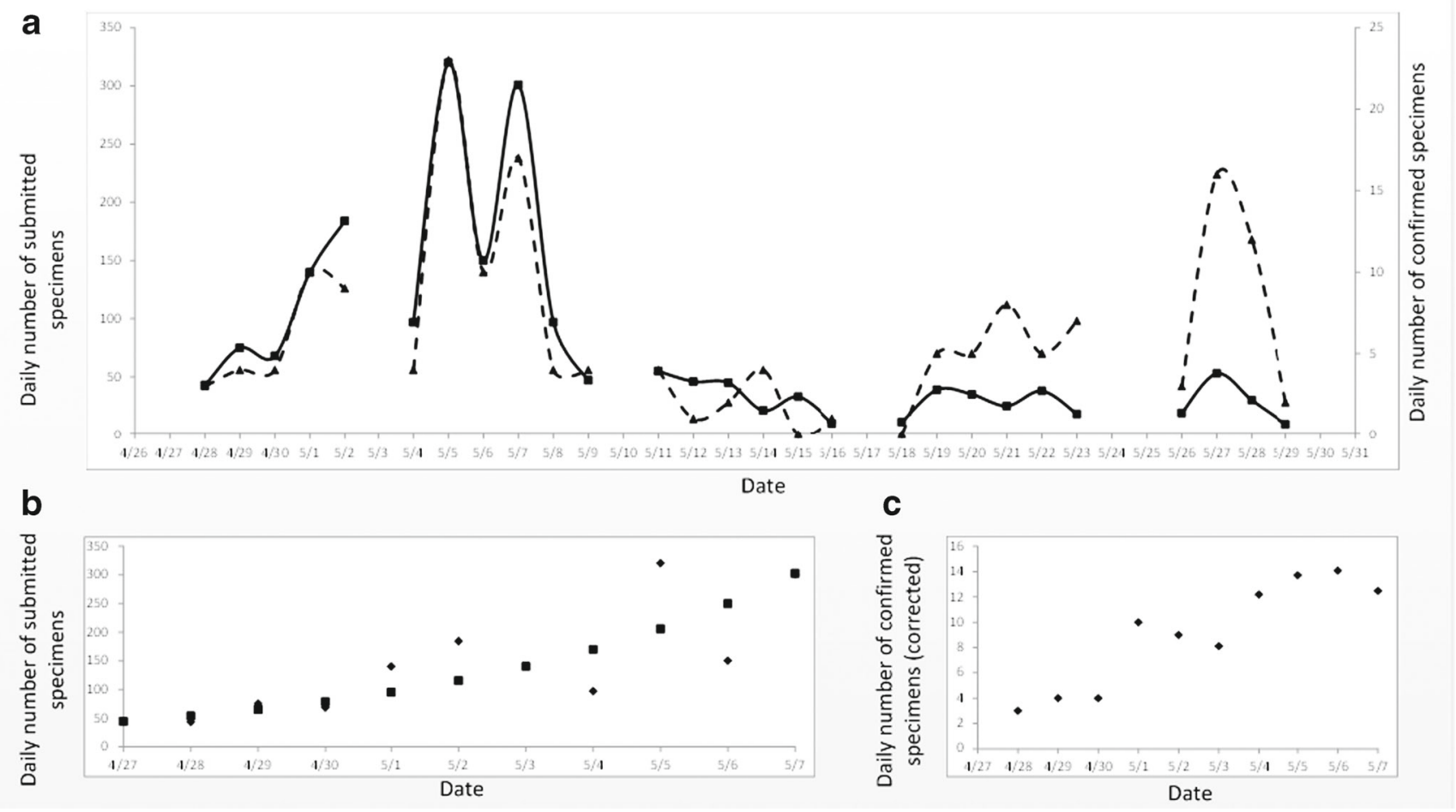

Fig. 3 a The dotted line shows the trend on the daily number of specimens submitted to BOL for confirmatory testing, from April 28th 2009, where the first suspected specimens were submitted, until May 29 th 2009, corresponding to the end of the first pandemic wave. The solid line shows the trend of cases confirmed with the pandemic H1N1

some of the specimens that were not recorded during Sundays, are confounded with the specimens of the upcoming week. Therefore, the curve corrects the absence of submitted specimens for Sunday, May 3rd, and the likely excess of recorded submission during the week beginning on May 4th.

With the fitted series of submitted specimens $X_{t}$, we inferred a corrected series of confirmed specimens $Y_{t}$ with the following Bayesian expression:

$p\left(\mathscr{Y}_{t}, X_{t}, \mathscr{E}_{t}\right)=p\left(\mathscr{Y}_{t} \mid X_{t}, \mathscr{E}_{t}\right) * p\left(\mathscr{E}_{t}\right)$

where $\mathscr{Y}_{t}$ corresponds to the random variable for $Y_{t}$, and $\mathscr{Y}_{t} \sim \operatorname{bin}\left(X_{t}, \mathscr{E}_{t}\right)$. Also, $\mathscr{E}_{t}$ is the random variable for $e_{t}$, the proportion of submitted specimens that result in confirmed cases. When $t \neq 6, \mathscr{E}_{t}=e_{t}$. When $t=6, \mathscr{E}_{t} \sim$ $N(0.058,0.012)$. The mean and standard deviation for the normal distribution are obtained from the $e_{t}$ values available until May 7th. Using slice sampling to find the maximum posterior, the $Y_{t}$ shown in Fig. 3c was obtained.

Table 7 shows the $r$ and $\widetilde{R}$ values obtained from the outbreak data. The fitting of the $r$ was initiated at the seventh outbreak day (May 4th). The $r$ for each day was fitted with a trend that included all the outbreak days up to the date. The values for $r$ and $\widetilde{R}$ were calculated using the "R0" package virus. b The diamond shaped dots represent the real trend of submitted specimens during the growth phase of the outbreak, which occurred from April 28th to May 7th. The squared shaped dots represent the series fitted through poisson regression. c Daily trend of confirmed cases corrected using the fitted trend of specimens submitted

in the R software, choosing the method proposed in Eq. 11 [48].

The values in Table 7 were obtained using a gamma shaped viral shedding profile $\left(\frac{E\left[\mathscr{V}_{t}^{S}\right]}{E\left[\sum_{t=0}^{\infty} \mathscr{V}_{t}^{S}\right]}\right)$ with a mean disease generation time of 2.3 days and a standard deviation of 0.68 days. The shape and parameters for the profile were fitted using the data in Table 3. We note that the parameter $\widetilde{R}$ is sensitive to the assumption of the viral shedding profile. Table 8 shows the variations in the value of $\widetilde{R}$ as the mean of the profile is increased, and the standard deviation is kept constant at 1 day.

The values for $\widetilde{R}$ were incorporated in our proposed epi model. We used our baseline model in Eq. 12, where the outbreak is only of PI, all infected cases were considered symptomatic and differences in the contact groups were

Table 7 Estimated values for $r$ and $\widetilde{R}$

\begin{tabular}{llll}
\hline Date & r & $\widetilde{R}$ & CI for $\widetilde{R}$ \\
\hline 4-May, 2009 & 0.266 & 2.1 & $(1.45,2.99)$ \\
5-May, 2009 & 0.243 & 1.9 & $(1.47,2.60)$ \\
6-May, 2009 & 0.212 & 1.8 & $(1.42,2.26)$ \\
7-May, 2009 & 0.174 & 1.6 & $(1.34,1.97)$ \\
\hline
\end{tabular}


Table 8 Values of $\widetilde{R}$ with varying mean disease generation time, $E[\mathscr{T}]$

\begin{tabular}{lll}
\hline$E[\mathscr{T}]$ (days) & $\widetilde{R}$ & CI for $\widetilde{R}$ \\
\hline 2.5 & 1.84 & $(1.45,2.35)$ \\
3.1 & 2.09 & $(1.57,2.80)$ \\
3.7 & 2.37 & $(1.69,3.36)$ \\
4.3 & 2.69 & $(1.83,4.02)$ \\
4.9 & 3.06 & $(1.98,4.81)$ \\
\hline
\end{tabular}

ignored. The accuracy of this model has been discussed in Section 4.1. Figure 4 a shows the outbreak recreated using $\widetilde{R}=1.6$ as an input to the model (i.e., $R_{o}=\widetilde{R}=1.6$ ). The whole time series reproduces the baseline behavior of disease spread in the absence of any containment or intervention initiated by any person or governmental institution. Therefore, the baseline and the real values for the daily cases from BOL data could not be compared directly, since the latter values occur under many layers of containment and mitigation.

The baseline presented in Fig. 4 includes two major components: The first is the trend of the time series, which is modulated by our proposed epi model. The second is the outbreak scale, which is modulated by the population network in the hosting $\mathrm{AB}$ model. In what follows, we provide evidence that our proposed epi model reproduces the trend observed in the real data. Figure $4 \mathrm{~b}$ shows the first ten days of exponential growth in the simulated data. Once a rate $r$ is obtained from these ten days and used in Eq. 11, the resulting $\widetilde{R}$ is 1.57 with CI $(1.38,1.78)$. This CI is within the one obtained for the $\widetilde{R}$ calculated for the first ten days in the BOL dataset (see Table 7, 7-May). We conclude that our epi model is able to capture the tendency of spread during the first stage of a nascent outbreak.

Any mitigation or containment can be incorporated to the presented outbreak baseline. As an example, previous work in the literature has demonstrated that incorporating voluntary household quarantine helps reducing the daily incidence and extends the duration of an outbreak. Additional measures like voluntary individual isolation, antiviral administration, and contact tracing will further reduce the daily incidence and increase the outbreak duration [58]. It is worth noting that additional modeling assumptions on the demographic and social behavioral components are highly driven by the strategies that the $\mathrm{AB}$ model will be testing (e.g. testing containment within the perimeter of an infected household cases, would at least require the geographic coordinates of the all the locations in a region). Such assumptions should be defined by consensus between the decision maker and the modeler [50].

\subsection{Effect of additional considerations in the accuracy of the epi model}

This case considers an outbreak of PI and SI, asymptomatic cases, and differences in the contact groups. Hence, new infections are considered to be guided by the rules in Table 1, and Eqs. 14, 15, 16, and 17.

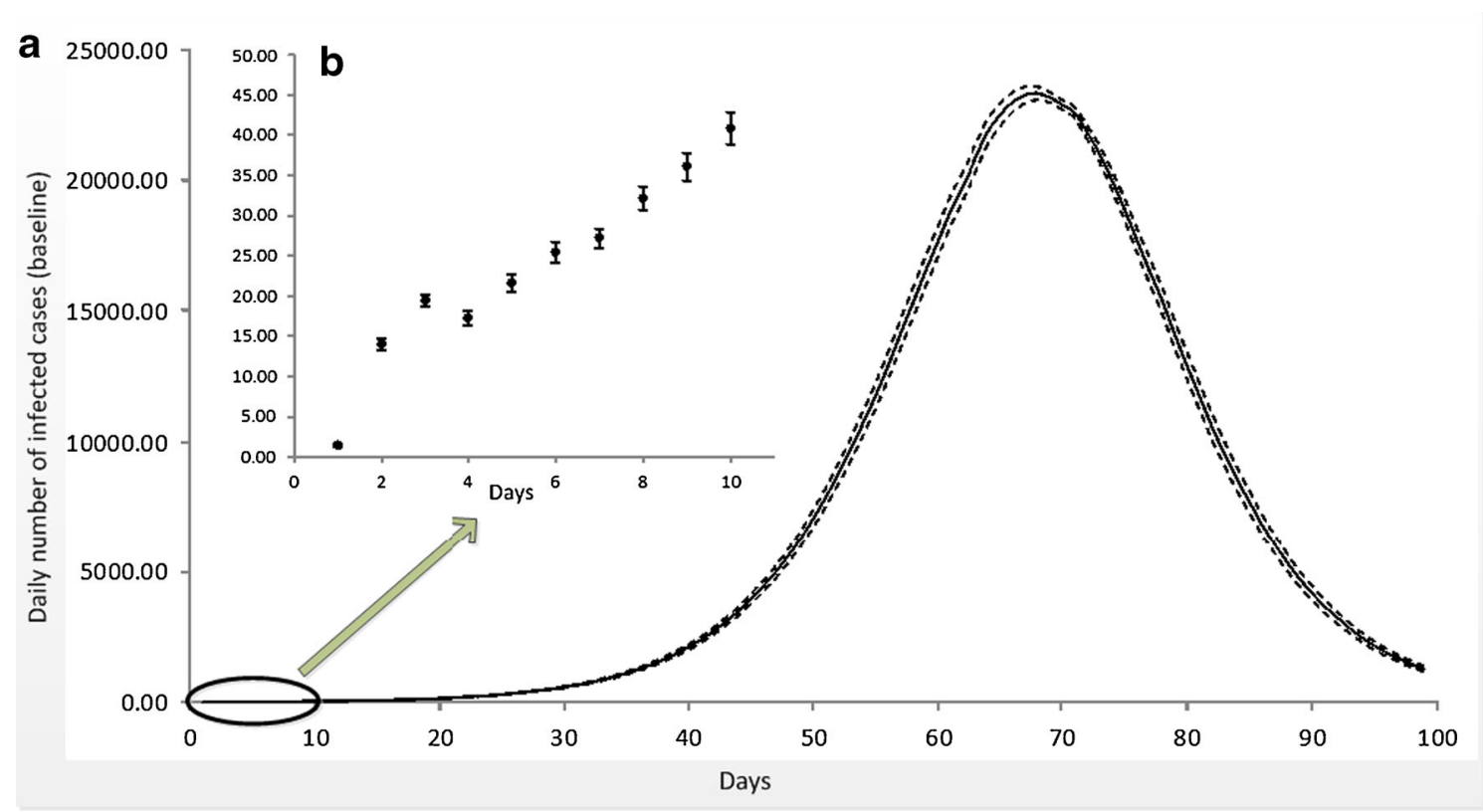

Fig. 4 a Baseline daily incidence as reproduced by the AB simulation model, using the proposed epi model. b A close-up of the figure showing the first 10 simulated days 
This scenario is set with a pandemic outbreak with $R_{o}=$ 1.8 , a seasonal outbreak with $R_{o}=1.3$, and assuming no cross-immunity (i.e., all the $\varepsilon_{j}^{l}$ factors are equal to 1 ). For 100,000 inhabitants and 146 replicates, the average $\widehat{R}_{O}$ for PI was found to be 1.67 with CI $(1.6432,1.6948)$, and the average $\widehat{R}_{o}$ for SI was found to be 1.39 with CI $(1.3633,1.4251)$. For the pandemic outbreak, the $\widehat{R}_{o}$ value is lower than the target $R_{o}$. We believe this reduction results from the assumption that interactions within households are higher than those in workplaces/schools and other places $\left(\kappa^{w}, \kappa^{o}<1.0\right.$ in Eqs. $14,15,16$ and 17. The households are the contact groups with less members, and as an increased number of members are infected and trying to infect each other, many attempts of new infections are failed, reducing the incidence. It is then observed that additional considerations might affect the accuracy of our basic epi model.

Figure 5a shows the disease spread of both the pandemic and the seasonal outbreaks in a replicate expressing the $\widehat{R}_{O}$ in generation zero. The pandemic outbreak infects up to
Fig. 5 a A typical replicate showing an outbreak of pandemic $\mathrm{H} 1 \mathrm{~N} 1\left(\widehat{R}_{O}=1.8\right)$ and seasonal H1N1 $\left(\widehat{R}_{o}=1.3\right)$, both expressed in generation zero. $\mathbf{b}$ Expected reproduction number per generation for for the scenario in $\mathbf{a}$

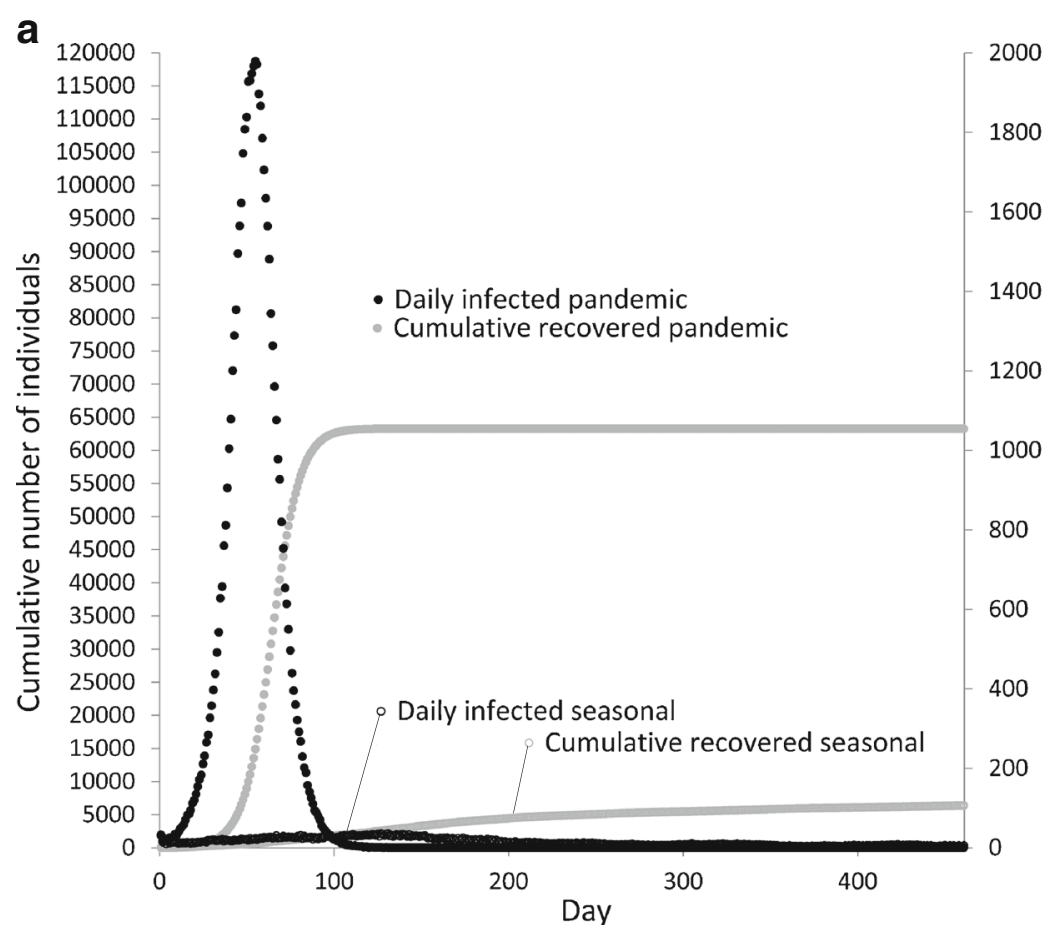

b

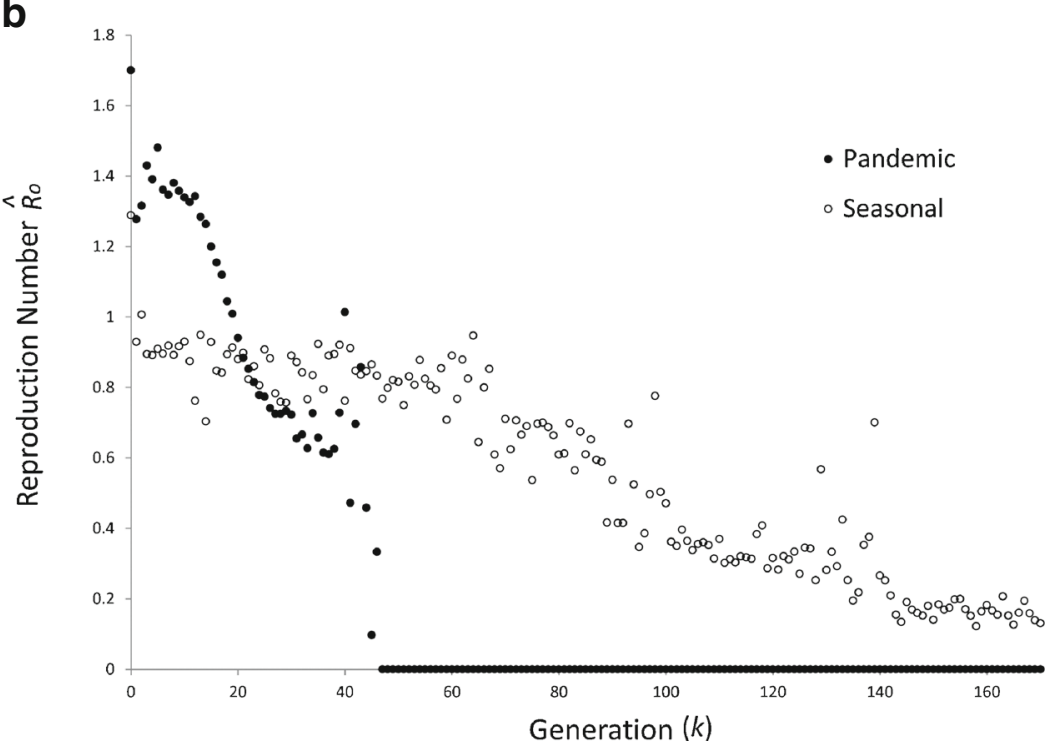


1966 individuals per day at its peak compared to only 36 for seasonal. The pandemic outbreak lasts until day 136 whereas the seasonal outbreak subsided around day 500. This is a reasonable incidence pattern of influenza viruses with low transmissibility. Figure 5b shows the behavior of the expected reproduction number. The expected reproduction number fluctuates over many generations until the seasonal outbreak disappear after 170 generations. The fluctuations are attributed to the small amount of infected cases in the last outbreak stages.

\section{Conclusion}

Operational modeling of viral infectious diseases is an emerging field that attempts to answer the following question: How can public health officials make model-based outbreak management decisions in less than 4-6 h?

To answer this question, several considerations must to be made, ranging from the data generation and collection, until the model set-up, model execution, and deployment of results. In this paper, we provide a solution to facilitate the set-up of AB simulation models, which are useful to provide decision support in the context of pandemic influenza. Our solution aims to make the $\mathrm{AB}$ models more useful for realtime applications, by reducing the complexity of calibrating the underlying epi model, and facilitating its interpretability.

In this paper, we have presented an epi model that reduces the computational complexity of the calibration process to a few operations that are automatically executable while the model is running. Our methodology allows to automatically ascertain that the $R_{O}$ introduced is the same $\widehat{R}_{O}$ obtained at the end of a simulation replicate, and eliminates iteration for fitting the internal model parameters. We have also demonstrated how the AB simulation model (supported by the proposed epidemiological model) can recreate projections that match the tendencies observed in the early stages of a real outbreak.

We showed that the complexity achieved by our approach is lower than any other iterative procedure used for $A B$ models. We note, however, that our epi model relaxes assumptions of irregular viral shedding and contact duration to achieve such complexity.

In the following, we state some of the model limitations and potential research directions.

The discussion of complexity was only based on the calibration algorithm, and excluded other components of the $\mathrm{AB}$ model such us simulation of agents and contacts. These components would certainly add to the complexity of the AB model, and would scale the simulation time with the population size. Calibration complexity and AB model scalability are two different issues affecting timely decision making by the public health policy makers. The whole focus of the paper was to address the first issue.

The calibration approach can be used at the beginning of a regional influenza emergency. However, it is not yet capable of updating the epidemiological parameters of the model during the outbreak progression. We plan to investigate the data requirements and modifications of our model to be able to periodically update model parameters.

The epidemiological model can be extended to accomodate co-circulation of two influenza viruses within a season. Such co-circulation might result in events of single infection, co-infection and re-infection, as it has occurred in previous pandemics [35]. Within the human body, interacting viruses can elicit cross-immune responses due to the reaction of $T$ cells and antibodies [20, 31, 54]. We considered such knowledge in our model by means of the cross-immunity factors described in Table 1, and we demonstrated that, when both viruses are simultaenously seeded in the population, variations in the cross immunity factors do not affect the calibration of the basic reproduction number (see the Appendix A for details).

Although we included this factors in our model, we found no evidence of research that evaluates cross-immunity when two viruses have been inoculated very close in time. Most of the cross immunity research is done within the framework of vaccine development, where the assessment of immunity estimates occurs weeks after the inoculation event, to observe the overall effectiveness of a possible vaccine response. Current research on modeling co-circulating viruses focuses on conditions of co-existence during several seasons, and they assume that the cross-immunity built during a season is mainly negligible $[46,49]$. Hence, we believe that there is a gap of knowledge in understanding the shortterm evolution of cross-immunity, and its true impact in the population.

A final limitation is that the epidemiological model does not consider alterations in the contact patterns as a behavioral response to disease spread. If behavioral responses are introduced in the model (e.g., when newly infected cases self-impose absenteeism), the effect is significant in reducing the simulated reproduction number and the accuracy of the calibration approach (Appendix B).

Acknowledgments The authors would like to thank Dr. Lillian Stark, retired virologist from the Florida Bureau of Laboratories, for providing aggregated counts of pandemic submitted and confirmed specimens. In addition, we thank Peter Holvenstot for running part of the model replicates used in our analysis. The authors appreciate the comments provided by the reviewers for the improvement of the manuscript. 


\section{Appendix A: Effect of cross immunity in the accuracy of the calibration approach}

We examined the effect of cross immunity when two influenza viruses co-circulate in a population by means of re-infection or co-infection.

The cross-immunity was implemented in the epidemiological model by the following factors:

$\varepsilon_{P I / S I}^{s}$ : factor by which pandemic viral shedding is affected by the cross-immunity of a seasonal virus. Both viruses infected the host simultaneously.

$\varepsilon_{S I / P I}^{s}$ : factor by which seasonal viral shedding is affected by the cross-immunity of a pandemic virus. Both viruses infected the host simultaneously.

$\varepsilon_{S I / P I}^{r}$ : factor by which pandemic viral shedding is affected by the cross-immunity of a seasonal virus that has already passed.
$\varepsilon_{S I / P I}^{c}$ : factor by which pandemic viral shedding is affected by the cross-immunity of a seasonal virus when both are occurring at the same time.

$\varepsilon_{P I / S I}^{r}$ : factor by which a seasonal viral shedding is affected by the cross-immunity of a pandemic virus that has already passed.

$\varepsilon_{P I / S I}^{c}$ : factor by which a seasonal viral shedding is affected by the cross-immunity of a pandemic virus when both are occurring at the same time.

We developed an experiment, where we simulated two viruses, a highly transmissible pandemic virus $\left(R_{o}^{P I}=1.8\right)$, and a mildly transmissible seasonal virus $\left(R_{o}^{S I}=1.3\right)$. Neither asymptomatic cases nor differences in the contact groups were included in the experiment.

We considered two levels for each of the cross immunity factors.
Fig. 6 a Reproduction number per generation (90\% individuals symptomatic), b Reproduction number per generation (70\% individuals symptomatic)
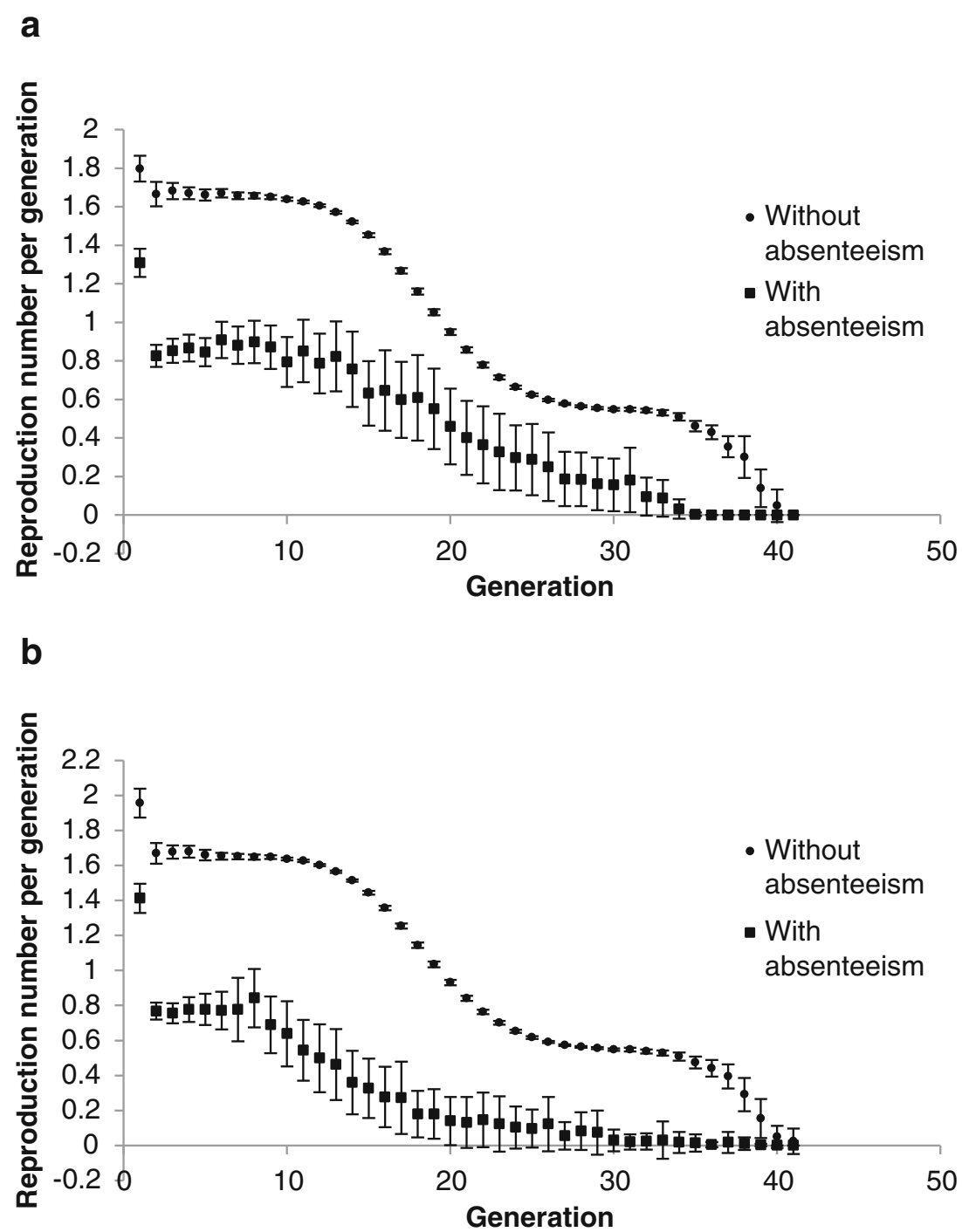
In the low level, the viral shedding profile of the virus generating the cross-immune response was $1.08 * \log 10$ times higher than the profile of the affected virus.

In the high level, the viral shedding profile of the virus generating the cross-immune response was $2 * \log 10$ times higher than the profile of the affected virus.

We ran 30 replicates for each the 64 factor combinations. In each of the factor combinations the confidence intervals for the final reproduction values were $(1.69,1.79)$ and $(1.34$, 1.40) for the pandemic and seasonal viruses respectively. We did not observe any difference in the final reproduction values as a consequence of varying the cross-immunity factors.

We then conclude that the cross-immunity factors do not influence the accuracy of the epi model based calibration approach.

In the model, both viruses were simultaneously seeded in the population, and the initially seeded pandemic cases were different from the initially seeded seasonal cases. At the beginning of the simulation, the entire simulation is susceptible to both viruses. At this point, the basic reproduction numbers were not affected by crossimmunity.

As the simulation progresses, more individuals have been infected with one virus and develop cross-immunity to a possible upcoming virus. At this stage, changes in the reproduction numbers are observed.

As future work, is of our interest to investigate and quantify the effect of the cross-immunity factors in the reproduction numbers, and the outbreak final size. In addition, we will consider the analysis of cocirculating viruses that are seeded in different periods of time.

\section{B: Effect of contact pattern alterations in the accuracy of the calibration approach}

In this experiment we simulated a population of 100000 individuals, and an $R_{O}=1.8$. In addition, we considered two levels for the percentage of symptomatic individuals in the simulation (i.e., $90 \%$ and $70 \%$ ).

The epidemiological model does not consider alterations in the contact patterns as a behavioral response to disease spread. To investigate the effect of contact alterations, we introduced self-imposed absenteeism in the model as a behavioral response, where a newly infected case will only contact her household members during the days of infection.

Figure $6 \mathrm{a}$ and $\mathrm{b}$ show the effect of self-imposed absenteeism. For both percentage levels of symptomatic individuals, absenteeism significantly reduces the reproduction number. This is a reasonable pattern since the symptomatic cases will initially contact and infect their household members, and the cases will not be able to express their disease reproduction pattern in other contacts. Many opportunities for infection are wasted by every case, and the reproduction numbers reduce. As indicated by the wide ranges in the confidence intervals when absenteeism occurs, there is additional variability inserted into the simulation because the number of people in each generation is small and contacts are irregular.

\section{References}

1. Google flutrends. http://www.google.org/flutrends/

2. Influenzanet. http://www.influenzanet.eu

3. Interim pre-pandemic planning guidance: community strategy for pandemic influenza mitigation in the united states. http://www. pandemicflu.gov/plan/community/communitymitigation.pdf

4. Modeling community containment for pandemic influenza: a letter report. http://www.nap.edu/catalog/11800.html

5. U.S. synthesized population 2005-2009 Version 1.0. https://www. epimodels.org/midas/pubsyntdata1.do

6. Study to determine the requirements for an operational epidemiological modeling process in support of decision making during disaster medical and public health response operations. Tech. rep., Yale New Haven Center for Emergency Preparedness and Disaster Response and US the Northern Command (2012)

7. Workgroup for identifying key components of a successful operational epidemiological modeling process, organized by the Yale New Haven Center for Emergency Preparedness and Disaster Response, and the US Northern Command (2012). Personal discussion with modelers and members of state and national public health agencies

8. CDC symposium on engineering and operations research in public health (2013). Personal discussion with CDC personnel

9. Ciofi degli Atti M, Merler S, Rizzo C, Ajelli M, Massari M, Manfredi P, Furlanello C, Scalia Tomba G, Iannelli M (2008) Mitigation measures for pandemic influenza in italy: an individual based model considering different scenarios. Plos One 3(3):1-11

10. Bajardi P, Poletto C, Ramasco J, Tizzoni M, Colizza V, Vespignani, A (2011) Human mobility networks, travel restrictions, and the global spread of 2009 H1N1 pandemic. PLos One 6(1):1-8

11. Balcan D, Colizza V, Goncalves B, Hu H, Ramascob J, Vespignani A (2010) Multiscale mobility networks and the spatial spreading of infectious diseases. PNAS 106(51):21484-21489

12. Balcan D, Hu H, Goncalves B, Bajardi P, Poletto C, Ramasco J, Paolotti D, Perra N, Tizzoni M, Van den Broeck W, Colizza V, Vespignani A (2009) Seasonal transmission potential and activity peaks of the new influenza $\mathrm{A}(\mathrm{H} 1 \mathrm{N1})$ : A Monte Carlo likelihood analysis based on human mobility. BMC Med 7(45), pp 1-12

13. Bisset K, Chen J, Feng X, Kumar A, Marathe M (2009) Epifast: a fast algorithm for large scale realistic epidemic simulations on distributed memory systems. In: Proceedings of 23rd international conference on supercomputing, pp 430-439

14. Brooks-Pollock E, Tilston N, Edmunds W, Eames K (2011) Using an online survey of healthcare-seeking behaviour to estimate the magnitude and severity of the $2009 \mathrm{~h} 1 \mathrm{n} 1 \mathrm{v}$ influenza epidemic in england. BMC Infect Dis 11(68), pp 1-8

15. Carrat F, Luong J, Lao H, Sallé AV, Lajaunie C, Wackernagel $\mathrm{H}$ (2006) A ‘small-world-like' model for comparing interventions 
aimed at preventing and controlling influenza pandemics. BMC Infect Dis 4(26), pp 1-14

16. Carrat F, Pelat C, Levy-Bruhl D, Bonmarin I, Lapidus N (2010) Planning for the next influenza H1N1 season: a modelling study. BMC Infect Dis 10(301), pp 1-9

17. Carrat F, Vergu E, Ferguson N, Lemaitre M, Cauchemez S, Leach S (2008) Timelines of infection and disease in human influenza: a review of volunteer challenge studies. Am J Epidemiol 167:775785

18. Chowell G, Miller M, Viboud C (2008) Seasonal influenza in the United States, France, and Australia: transmission and prospects for control. J R Soc Interface 4:155-156

19. Chowell G, Nishiura H, Bettencourt LM (2007) Comparative estimation of the reproduction number for pandemic influenza from daily case notification data. J Infect 62:200-203

20. Corti D, Voss J, Gamblin S, Codoni G, Macagno A, Jarrossay D, Vachieri S, Pinna D, Minola A, Vanzetta F, Silacci C, Fernandez B, Agatic G, Bianchi S, Giacchetto I, Calder L, Sallusto F, Collins P, Haire L, Temperton N, Langedijk J, Skehel J, Lanzavecchia A (2011) A neutralizing antibody selected from plasma cells that binds to group 1 and group 2 influenza A Hemagglutinins. Science 333:850-856

21. Das T, Savachkin A (2008) A large scale simulation model for assessment of societal risk and development of dynamic mitigation strategies. IIE Trans 40(9):893-905

22. Diekmann O, Heesterbeek J (2000) Mathematical epidemiology of infectious diseases: model building, analysis and interpretation, Wiley, New York

23. Ferguson N, Cummings D, Fraser C, Cajka J, Cooley P, Burke D (2006) Strategies for mitigating an influenza pandemic. Nature 442(27):448-452

24. Ferguson N, Cummings DA, Cauchemez S, Fraser C, Riley S, Aronrag M, Lamsirithaworn S, Burke D (2005) Strategies for containing an emerging influenza pandemic in Southeast Asia. Nature 437(7056):209-214

25. Flahault A, Vergu E, Boëlle P (2009) Potential for a global dynamic of influenza a (h1n1). BMC Infect Dis 9(129):1-11

26. Fraser C, Donnelly C (2009) Pandemic potential of a strain of influenza A (H1N1): early findings. Science 324:1557-1561

27. Germann T, Kadau K, Longini I, Macken C (2006) Mitigation strategies for pandemic influenza in the United States. PNAS 103:5935-5940

28. Ginsberg J, Mohebbi M, Patel R, Brammer L, Smolinski M, Brilliant, L (2009) Detecting influenza epidemics using search engine query data. Nature 457(19):1012-1015

29. Glass R, Beyeler W, Min H (2006) Targeted social distancing design for pandemic influenza. Emerg Infect Dis 12(11):16711681

30. Gojovic M, Sander B, Fisman D, Krahn M, Bauch C (2009) Modelling mitigation strategies for pandemic (H1N1) 2009. CMAJ 181(10):673-680

31. Guo H, Santiago F, Lambert K, Takimoto T, Topham D (2011) T cell-mediated protection against lethal 2009 pandemic H1N1 influenza virus infection in a mouse model. J Virol 85(1):448455

32. Halder N, Kelso J, Milne G (2010) Developing guidelines for school closure interventions to be used during a future influenza pandemic. BMC Infect Dis 10(221), pp 1-14

33. Halloran M, Ferguson N, Longini I, Macken C (2008) Modeling targeted layered containment of an influenza pandemic in the United States. PNAS 105(12):4639-4644
34. Kenah E, Chao D, Matrajt L, Halloran M, Longini I (2011) The global transmission and control of influenza. PLoS One 6(5):1-10

35. Liu W, Li Z, Tang F, Wei M, Tong Y, Zhang L, Xin Z, Ma M, Zhang X, Liu L, Zhan L, He C, Yang H, Boucher C, Hendrik J, Cao W (2010) Mixed infections of pandemic H1N1 and seasonal H3N2 viruses in one outbreak. Clin Infect Dis 50(10):13591365

36. Longini I, Halloran M, Nizam A, Yang Y (2004) Containing pandemic influenza with antiviral agents. Am J Epidemiol 159(7):623-633

37. Longini I, Nizam A, Shufu X, Ungchusak K, Hanshaoworakul W, Cummings D, Halloran M (2005) Containing pandemic influenza at the source. Science 309:1083-1087

38. Meloni S, Perra N, Arenas A, Gomez S, Moreno Y, Vespignani A (2011) Modeling human mobility responses to the large-scale spreading of infectious diseases. Sci Rep 1(62):1-7

39. Merler S, Ajelli M (2010) The role of population heterogeneity and human mobility in the spread of pandemic influenza. Proc $\mathrm{R}$ Soc B 277:557-565

40. Mills C, Robins J, Lipsitch M (2004) Transmissibility of 1918 pandemic influenza. Nature 432:904-906

41. Mossong J, Hens N, Jit M, Beutels P, Auranen K, Mickolajczyk R, Massari M, Salmaso S, Scalia Tomba G, Wallinga J, Heijne J, Sadkowska-Todys M, Rosinska M, John Edmunds M (2008) Social contacts and mixing patterns relevant to the spread of infectious diseases. PLoS Med 5(3):381-391

42. Nelson M, Spiro D, Wentworth D, Beck E, Jiang F, Ghedin E, Halpin R, Bera J, Hine E, Proudfoot K, Stockwell T, Lin X, Griesemer S, Swati K, Bose M, Viboud C, Holmes E, Henrickson K (2009) The early diversification of influenza a/h1n1pdm. PLoS Curr 1:RRN1126

43. Nelson M, Tan Y, Ghedin E, Wentworth D, St. George K, Edelman, L, Beck E, Fan J, Tsan-Yuk LT, Kumar S, Spiro D, Simonsen L, Viboud C, Holmes E, Henrickson K, Musser J (2010) Phylogeography of the spring and fall waves of the H1N1/09 pandemic influenza virus in the united states. J Virol 85(2):828834

44. Nsoesie E, Marathe M, Brownstein J (2013) Forecasting peaks of seasonal influenza epidemics. Plos current outbreaks

45. Nuno M (2010) Pandemic influenza: potential contribution to disease burden. In: Preedy V, Watson R (eds) Handbook of disease burdens and quality of life measures. Springer, New York

46. Nuno M, Feng Z, Martcheva M, Castillo-Chavez C (2005) Dynamics of two-strain influenza with isolation and partial crossimmunity. SIAM 65(3):964-982

47. Oakley J, O'Hagan A (2004) Probabilistic sensitivity analysis of complex models: a Bayesian approach. J R Stat Soc B 66(3):751769

48. Obadia T, Haneef R, Boëlle P (2012) The R0 package: a toolbox to estimate reproduction numbers for epidemic outbreaks. BMC Med Inf Decis Making 12(147), pp 1-9

49. Omori R, Adams B, Sasaki A (2010) Coexistence conditions for strains of influenza with immune cross-reaction. J Theor Virol 262:48-57

50. Prieto D, Das T, Savachkin A, Uribe A, Izurieta R, Malavade S (2012) A systematic review to identify areas of enhancements of pandemic simulation models for operational use at provincial and local levels. BMC Publ Health 12(251), pp 1-13

51. Rahmandad H, Sterman J (2008) Heterogeneity and network structure in the dynamics of diffusion: comparing agent-based and differential equation models. Manag Sci 54(5):998-1014 
52. Rosenfeld L, Fox C, Kerr D, Marziale E, Cullum A, Lota K, Stewart J, Thompson M (2009) Use of computer modeling for emergency preparedness functions by local and state health official: a needs assessment. J Public Health Manag Pract 15(2):96104

53. Syspal V, Hatzakis A (2009) School closure is currently the main strategy to mitigate influenza $\mathrm{A}(\mathrm{H} 1 \mathrm{~N} 1) \mathrm{v}$ : a modelling study. Eurosurveillance 14(24):1-6

54. Thomas P, Keating R, Hulse-Post D, Doherty P (2006) Cellmediated protection in influenza infection. Emerg Infect Dis 12(1):48-54

55. Uribe A, Savachkin A, Santana A, Prieto D, Das T (2011) A predictive decision aid methodology for dynamic mitigation of influenza pandemics: special issue on optimization in disaster relief. OR Spectrum 6 May, pp 1-36

56. Wallinga J, Lipsitch M (2007) How generation intervals shape the relationship between growth rates and reproduction numbers. Proc R Soc B 274:599-604

57. White L, Wallinga J, Finelli L, Reed C, Riley S, Lipsitch M, Pagano M (2009) Estimation of the reproductive number and the serial interval in early phase of the 2009 Influenza the current Influenza A/H1N1 pandemic in the USA. Influenza Other Respir Viruses 3(6):267-276

58. Wu J, Riley S, Fraser C, Leung G (2006) Reducing the impact of the next influenza pandemic using household-based public health interventions. PLoS Med 3(9):1532-1540 